\title{
1 An interplay of population size and environmental heterogeneity 2 explains why fitness costs are rare
}

3 Yashraj Chavhan ${ }^{1}$, Sarthak Malusare ${ }^{1,2}$, and Sutirth Dey ${ }^{1, *}$

$4{ }^{1}$ Indian Institute of Science Education and Research (IISER) Pune, Dr Homi Bhabha Road,

5 Pashan, Pune, Maharashtra, 411008, India.

$6 \quad{ }^{2}$ Present address: Gaia Doctoral School, Institut des sciences de l'évolution (ISEM) 1093-

71317 Route de Mende, 34090, Montpellier, France.

8 *Corresponding author: Sutirth Dey, Biology Division, Indian Institute of Science-Education 9 and Research Pune, Dr Homi Bhabha Road, Pune, Maharashtra 411 008, India.

Phone: +91-20-25908054. Email: s.dey@iiserpune.ac.in

Emails: yashraj.chavhan@students.iiserpune.ac.in (YC); sarthak.malusare@students.iiserpune.ac.in (SM)

\section{Author contributions}

YC and SD designed the study. YC and SM conducted the experiments. YC analysed the data.

Keywords: Costs of adaptation, whole-genome whole-population sequencing, mutation 


\section{Abstract}

23 Theoretical models of ecological specialization commonly assume that adaptation to one

24 environment leads to fitness reductions (costs) in others. However, empirical studies often fail

25 to detect such costs. We addressed this conundrum using experimental evolution with

26 Escherichia coli in several homogeneous and heterogeneous environments at multiple

27 population sizes. We found that in heterogeneous environments, smaller populations paid significant costs, but larger ones avoided them altogether. Contrastingly, in homogeneous environments, larger populations paid more costs than the smaller ones. Overall, large population sizes and heterogeneous environments led to cost avoidance when present together but not on their own. Whole-genome whole-population sequencing revealed that the enrichment of multiple mutations within the same lineage (and not subdivision into multiple distinct specialist subpopulations) was the mechanism of cost avoidance. Since the conditions revealed by our study for avoiding costs are widespread, it explains why the costs expected in theory are rarely detected in experiments. 
37 Costs of adaptation, also known as 'fitness costs' and 'true trade-offs,' entail that a fitness increase in one environment leads to a fitness decline in another (Bono et al. 2017). Such costs are instrumental in understanding why species tend to favour a particular set of environmental conditions over others (Fry 1996; Bono et al. 2017). Apart from answering such fundamental questions in evolutionary ecology, understanding fitness costs can also help in combating practical challenges like the rampant spread of antibiotic resistance(Andersson \& Hughes 2010) and forecasting how species would respond to climate change (Wallenstein \& Hall 2012). Although such costs are a fundamental assumption of numerous models of ecological specialization (Levins 1968; Futuyma \& Moreno 1988; Fry 1996), a large number of experimental evolution studies spanning diverse taxa have failed to detect them (Rausher 1984; Coustau et al. 2000; Vasilakis et al. 2009; Vila-Aiub et al. 2009; Friman \& Buckling 2013). Consequently, explaining this rarity of detectable fitness costs has been a major challenge for evolutionary studies over the last two decades (Joshi \& Thompson 1995; Fry 1996; Agrawal et al. 2010; Remold 2012).

Here we investigate the evolutionary emergence and avoidance of fitness costs in asexual microbial populations, which have proven to be convenient model systems for experimental evolution studies over hundreds of generations (Kassen 2014; Bono et al. 2017). Whereas numerous microbial experimental evolution studies have reported the absence of detectable fitness costs altogether, several others have found such costs in some microbial populations but not in others (see Table S1 for a detailed list).

An important but trivial explanation for the failure to find fitness costs is the absence of any real costs altogether (Coustau et al. 2000). Indeed, some recent investigations have found the pleiotropy of new mutations to be largely positive and not negative (i.e., costly) (Sane et al. 
2018). More importantly, the extant literature offers three distinct explanations as to why fitness costs may exist but remain undetected in empirical studies (Velicer \& Lenski 1999; Coustau et al. 2000). First, costs can be detected only under certain environmental conditions which the experimental setup may fail to provide (Coustau et al. 2000; Agrawal et al. 2010; Kassen 2014). Second, it is a statistically demanding task to detect negative pleiotropy (aka antagonistic pleiotropy), the very foundation of fitness costs, which entails that a mutation that is beneficial in one environment is deleterious in another. This is because the statistical significances of both the beneficial and deleterious effects need to be established simultaneously for detecting costs. If the experiment does not have enough statistical power to detect these opposite effects simultaneously, costs would not be detected (Coustau et al. 2000; Bono et al. 2017). Third, the emergence of fitness costs is expected to require a threshold amount of time; such costs may appear only after several thousand generations of microbial evolution have passed (Velicer \& Lenski 1999; Jasmin \& Zeyl 2013; Satterwhite \& Cooper 2015), and therefore would be detectable only in very long-term experimental evolution studies.

A recent meta-analysis of microbial experimental evolution studies provides a new explanation for the emergence of fitness costs based on environmental heterogeneity, suggesting that environments imposing a single (homogeneous) selection pressure frequently lead to fitness costs that can be avoided in heterogeneous environments (which fluctuate across multiple individual selection pressures) (Bono et al. 2017). Antagonistic pleiotropy can evolve freely if the environment does not allow the ensuing costs of adaptation to be expressed. Since selection would be blind to the antagonistic pleiotropic effects if the environment does not change, fitness costs are more likely to appear in homogeneous environments with a single selection pressure as compared to heterogeneous environments with multiple fluctuating selection pressures. 
Unfortunately, the above prediction holds only weakly as many microbial experimental evolution studies have failed to find lower costs in heterogeneous environments as compared to homogeneous ones (Jasmin \& Kassen 2007b; Presloid et al. 2008; Friman \& Buckling 2013; Ketola \& Saarinen 2015). This opens up the possibility that factors other than environmental heterogeneity may be important in shaping the emergence of fitness costs. One such factor is populations size, which has been shown to be important in shaping the correlated changes in populations' fitness in alternative environments (Chavhan et al. 2019a, 2020). For example, a recent study showed that larger populations evolving in a homogeneous environment containing a single carbon source suffer greater fitness costs in alternative environments (Chavhan et al. 2020). These results could be explained with a combination of two notions. First, adaptation in very large populations is primarily driven by beneficial mutations of large effect sizes (Desai \& Fisher 2007a; Chavhan et al. 2019b). Second, larger beneficial mutations are expected to carry heavier disadvantages in alternative environments (Lande 1983; Orr \& Coyne 1992).

Taken together, the extant literature suggests that environmental heterogeneity and population size are two important factors that can potentially shape the evolution of fitness costs. However, the effects of the interaction of these two factors remains unknown. Interestingly, this interaction can be expected to play out in two contrasting ways.

First, if mutational pleiotropy across environmental components is largely antagonistic, and large benefits in one context entail large costs in another, the multiplicity of selection pressures in a heterogeneous environment would prevent the enrichment of costly large effect mutations, even if the latter were accessible to the population. This is akin to Fisher's formulation of micromutationism where adaptation is expected to proceed via mutations of small effects(Fisher 1930). In this scenario, in heterogenous environments, both large and small populations are expected to pay similar costs. 
Second, in a heterogeneous environment, evolving in larger numbers can make populations stumble upon greater number of mutations that are beneficial in a given environmental state, but not necessarily in others. The presence of multiple mutations within an individual belonging to a large asexual population has the potential to offset the costs carried by individual mutations in isolation. In this scenario, adapting in larger numbers in a heterogeneous environment would lead to the avoidance of fitness costs. Interestingly, bacterial experimental evolution studies conducted in heterogeneous environments agree with this notion: studies on smaller populations tend to detect costs, while those using larger populations do not (Fig 1a).

Stated differently, in homogeneous environments, larger populations are expected to pay heavier costs of adaptation (Chavhan et al. 2020). However, in heterogeneous environments, larger populations may either pay similar or lower costs as compared to smaller populations, depending upon which one of the above two possibilities dominates the underlying adaptive dynamics. To the best of our knowledge, no studies in the existing literature have tested these contrasting expectations empirically.

Here we study how environmental heterogeneity and population size interact with each other to influence the evolutionary emergence or avoidance of fitness costs. To this end, we use experimental evolution with clonally derived Escherichia coli populations in both heterogeneous and homogeneous environments at different population sizes for $\sim 480$ generations. We investigate if population size has similar effects on fitness costs in homogeneous and heterogeneous environments. We also test if evolving in a heterogeneous environment can lead to cost avoidance, regardless of the population size. We show that population size influences costs in opposite ways in homogeneous and heterogeneous environments. Interestingly, large population size and heterogeneous environments lead to evolutionary avoidance of costs when present simultaneously but not in isolation. Mutational frequency distributions obtained by whole-genome whole-population sequencing revealed how 
environmental heterogeneity led to cost avoidance in large populations but not in smaller ones.

Based on these observations, we propose a new explanation for the rarity of fitness costs in made in the last two decades of microbial experimental evolution.

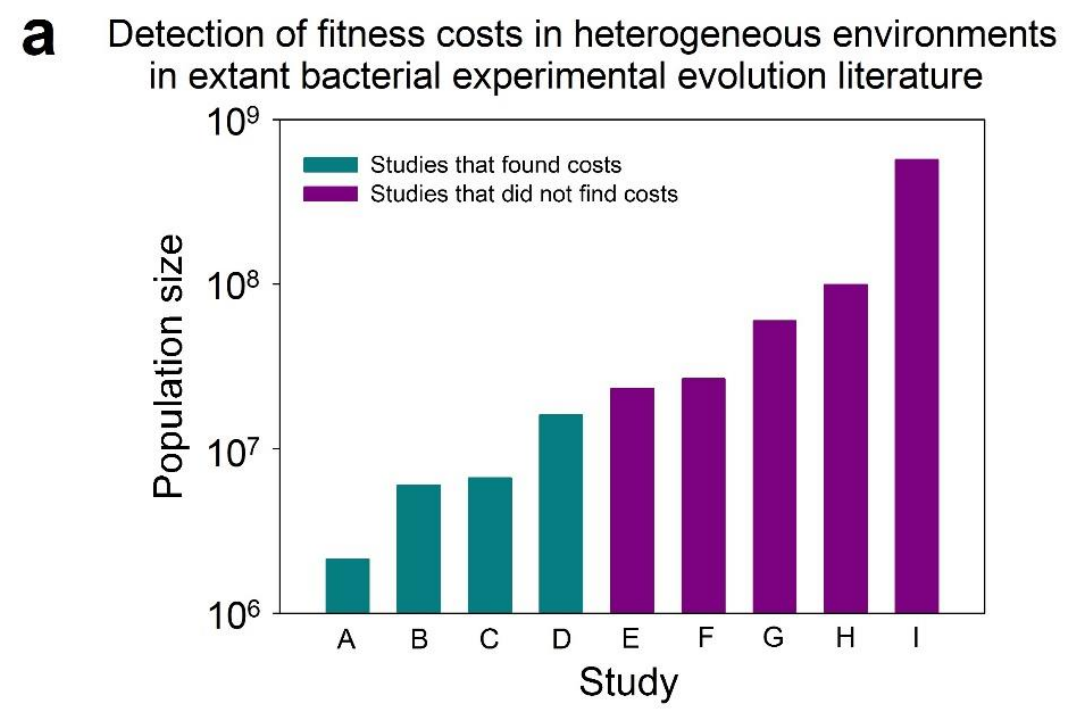

b Schematic representation of the experimental evolution in this study

139
Selection environment Heterogeneous (Fluctuating (F))

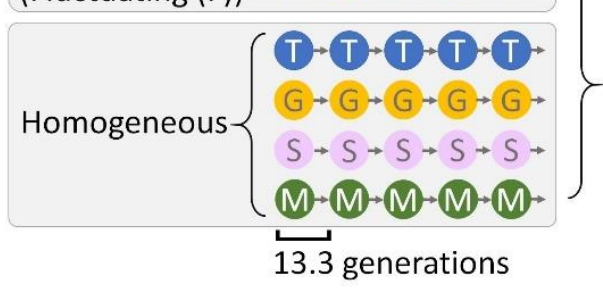

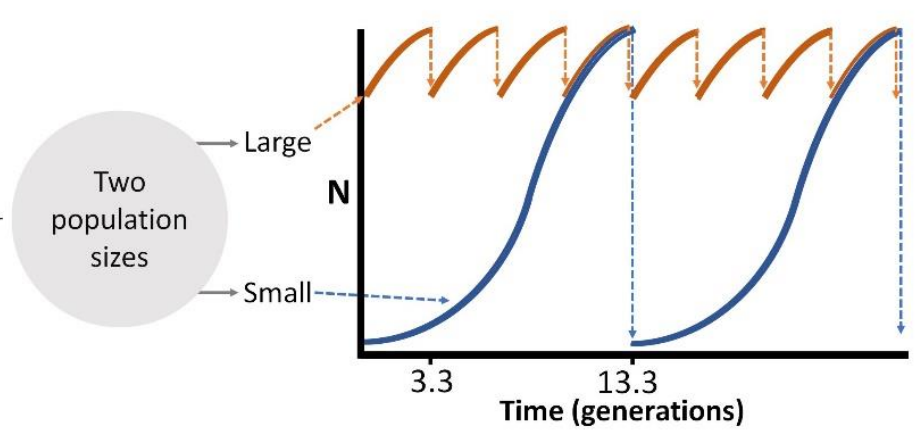

Fig. 1. (a) The harmonic mean sizes of laboratory populations in existing bacterial experimental evolution studies on fitness costs conducted in heterogeneous environments. See Supplementary Text (ST.1) for the details of the studies shown in the ordinate. (b) A schematic representation of our evolution experiment. The experimental populations were maintained in five distinct environments at two different population sizes. T, G, S, and M refer to thymidine, galactose, sorbitol, and maltose, respectively. $\mathrm{N}$ stands for absolute population size. In the heterogeneous (randomly fluctuating environment, the identity of the sole carbon source changed every 13.3 generations. See the text for further details. 


\section{Results and Discussion}

\section{Large population size and heterogeneous environments led to cost avoidance when present}

\section{together but not on their own}

We carried out experimental evolution with clonally derived E. coli populations in five different nutrient limited environmental conditions at two different population sizes for $\sim 480$ generations (Fig. 1b). This design gave rise to ten different evolutionary regimens (FL, FS, TL, TS, GL, GS, SL, SS, ML, and MS; where the first letter represents the sole carbon source in a regimen's selection environment (fluctuating $(F)$, thymidine $(T)$, galactose $(G)$, sorbitol $(S)$, and maltose (M)) while the second letter stands for its population size (L (large) and S (small)). The harmonic mean population size for our principal treatment (F, Fluctuating (heterogeneous) environment) was $\sim 1.01 \times 10^{8}$ for the large (FL) populations and $\sim 4.04 \times 10^{5}$ for the small (FS) populations. Moreover, the adaptively relevant population sizes for $\mathrm{L}$ and $\mathrm{S}$ in this treatment were approximately equal to $9.13 \times 10^{6}$ and $2.28 \times 10^{3}$, respectively (Chavhan et al. 2019b). In the FL and FS regimens, the identity of the sole carbon source fluctuated randomly across four distinct states (T, G, S, and M) approximately every $~ 13.3$ generations (Fig. 1b). Our study also involved four distinct homogeneous environmental controls, each with an unchanging identity of the sole carbon source corresponding to one of T, G, M, or S (Fig. 1b). With six replicates per regimen, our experiment involved 60 independently evolving populations in total. All the large (L) populations faced a periodic bottleneck ratio of 1:10 while all the small (S) populations experienced a periodic bottleneck of 1:104. We manipulated the timing and frequency of bottlenecks to ensure that large and small populations did not spend significantly different times in the stationary phase (Fig. 1b; see Methods for details).

We conducted growth measurements to obtain high-resolution growth curves for all the 60 independently evolving populations in all four distinct sole carbon sources (T, G, M, and S) at 

the end of the evolution experiment. We used the maximum growth rate $(\mathrm{R})$ as the measure of 173 fitness (Leiby \& Marx 2014; Karve et al. 2015; Chavhan et al. 2019a, b) (see Methods for

174 details). We identified the occurrence of significant costs of adaptation in our experimental 175 populations as cases that showed adaptation to one environment and simultaneous 176 maladaptation to another. To this end, we carried out single sample $t$-tests with the ancestral 177 fitness level (scaled to 1) as the reference value. We then corrected for family-wise error rates 178 using the Holm- Šidàk procedure (Abdi 2010). Cases with fitness $>1$ (corrected $P<0.05$ ) were 179 identified as adaptations; analogously, cases with fitness $<1$ (corrected $P<0.05$ ) were 180 identified as maladaptations.

181 We found that twenty-one out of the forty possible combinations of regimen and assay environment showed significant fitness changes as compared to the common ancestor (corrected $P<0.05$; see Table S2). We used this information to analyse the effects of two

184 factors that are expected to be important in shaping the evolution of fitness costs in bacterial populations, namely population size and environmental heterogeneity. 


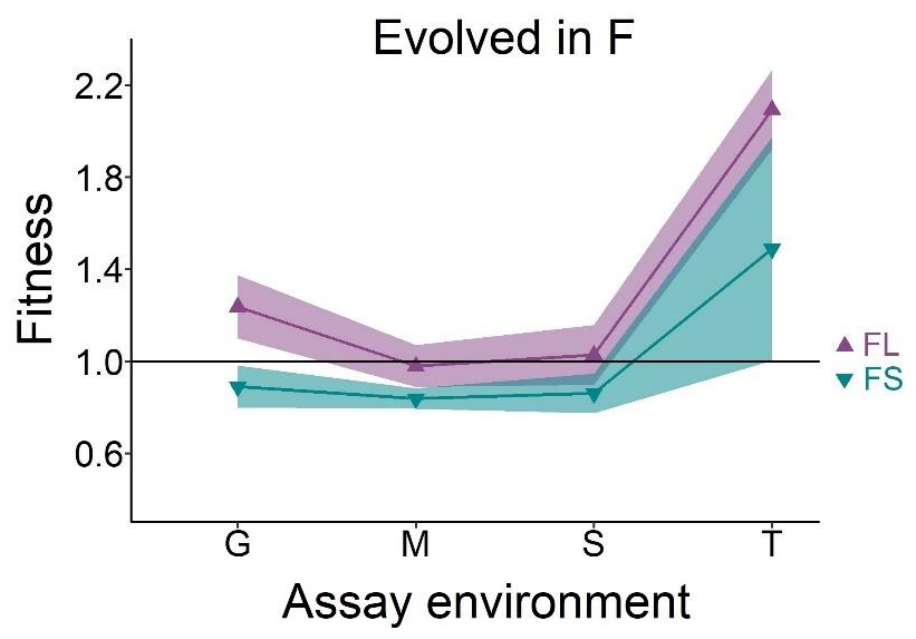

Fig. 2. Reaction norms of fitness of large (FL) and small (FS) populations evolved in the heterogeneous environment across the four environmental states faced during evolution. G, M, S, and T represent galactose, maltose, sorbitol, and thymidine, respectively. The error bands represent $95 \%$ CI ( $t$-distribution). The solid black line represents the ancestral fitness. FL adapted simultaneously to two environments ( $\mathrm{G}$ and $\mathrm{T})$ and avoided the costs of adaptation across all the environmental pairs under consideration. Contrastingly, FS adapted to T and paid costs of adaptation in the other three environments ( $\mathrm{G}, \mathrm{M}$, and S). See Tables S2 and S3 for detailed statistics.

In the heterogeneous (F) environment, the large populations (FL) completely avoided costs across all the environmental pairs under consideration (Fig. 2; Tables S2 and S3). FL adapted simultaneously to both $\mathrm{T}$ and $\mathrm{G}$ and did not show a significant change in fitness (vis-à-vis the common ancestor) in S and M (Fig. 2; Tables S2 and S3). On the other hand, the small populations evolved in the heterogeneous environment (FS) adapted only to T, becoming maladapted to (and hence paid a cost of adaptation in) the other three sole carbon sources $(\mathrm{G}$, S, and M) (Fig. 2; Tables S2 and S3). Taken together, when evolved in the heterogeneous (F) environment, the small populations paid greater costs than the large populations, with the latter avoiding all costs altogether. completely. Here, the large populations paid heavier costs of adaptation than the smaller ones 

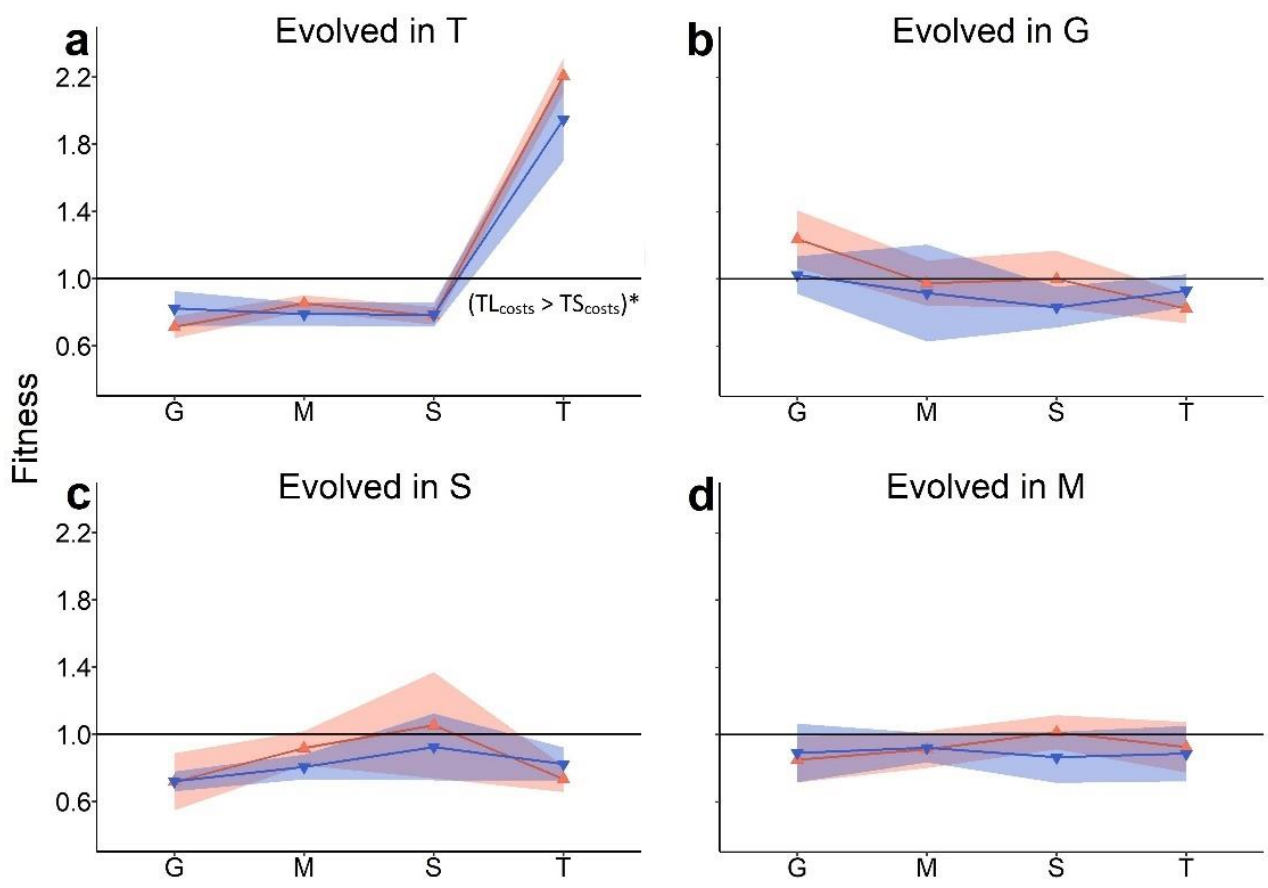

Large populations

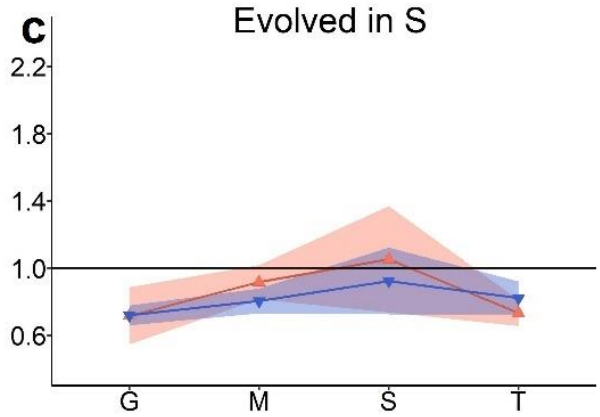

Small populations

Assay environment

Fig. 3. Reaction norms of fitness of populations evolved in homogeneous environments. $\mathrm{G}, \mathrm{M}, \mathrm{S}$, and T represent galactose, maltose, sorbitol, and thymidine, respectively. The error bands represent $95 \% \mathrm{CI}$ ( $t$-distribution). The solid black line represents the ancestral level of fitness. See Tables S2 and S3 for detailed statistics. (a) When evolved in T, both the large (TL) and small populations paid costs in the other three environments ( $\mathrm{G}, \mathrm{M}$, and $\mathrm{S})$. The costs paid by TL were significantly greater than those paid by TS (Chavhan et al. 2020). (b) GL paid significant costs in T. GL did not have significantly different fitness relative to the common ancestor in $\mathrm{M}$ and S. GS did not adapt significantly to G. Hence there were no costs of adaptation in this case. (c) Both SL and SS failed to show significantly different fitness with respect to the common ancestor. Hence there were no costs of adaptation in either SL or SS. (d) Neither ML nor MS had significantly different fitness with respect to the common ancestor. 

each other (Chavhan et al. 2020). In other words, adaptation to $\mathrm{T}$ is accompanied by maladaptation to G, and vice-versa (Chavhan et al. 2020). Agreeing with this notion, we found that when evolved in the heterogeneous environment (where the sole carbon source fluctuated randomly), the small populations (FS) indeed suffered from the T-G costs. Specifically, FS adapted to $\mathrm{T}$ but became significantly maladapted to G (Fig. 2; Table S3). Contrastingly, the large populations evolved in the heterogeneous environment (FL) completely bypassed the expected T-G trade-off, adapting simultaneously to both the carbon sources, thereby avoiding the costs of adaptation across this environmental pair (Fig. 2, Table S3).

Taken together, evolution in the ten regimens of our study reveals that an interplay of environmental heterogeneity and population size shaped how fitness costs evolved. We found that population size had opposite effects on costs of adaptation during evolution in heterogeneous versus homogeneous environments. While in homogeneous environments, larger populations evolved greater costs; contrastingly, in heterogeneous environments, smaller populations paid greater costs while larger ones avoided them altogether. Importantly, neither environmental heterogeneity nor population size could sufficiently explain the emergence (or avoidance) of costs on their own (compare Figs. 2 and 3). Overall, costs could be avoided altogether only when heterogeneous environments and large population size were present simultaneously (the FL regimen).

Conventional explanations cannot account for the avoidance of fitness costs in our experiments

249 Conventional notions about the rarity of detectable fitness costs failed to explain our observations. One such explanation is that perhaps the experiment did not provide the relevant 
conditions for costs to be expressed (Coustau et al. 2000; Agrawal et al. 2010; Kassen 2014).

This was not the case in our experiments as several environmental pairs showed significant costs of adaptation. Another potential explanation is that the substantial statistical demands of establishing antagonistic pleiotropy were not met (Coustau et al. 2000; Anderson et al. 2013; Ågren et al. 2013; Bono et al. 2017). However, we were able to statistically detect costs caused by antagonistic pleiotropy in multiple regimens and in both homogeneous and heterogeneous environments (Figs 2 and 3). Finally, an often-quoted explanation for the lack of fitness costs is the relatively short duration of the experimental evolution study (Velicer \& Lenski 1999; Jasmin \& Kassen 2007a; Jasmin \& Zeyl 2013; Satterwhite \& Cooper 2015; Schick et al. 2015). However, this was simply not true in our case, as several fitness costs had already emerged over the $\sim 480$ generations of selection.

As discussed earlier, evolution in heterogeneous environments is expected to lead to lower costs than evolution in homogeneous environments because the former offer multiple dynamic selection pressures (Bono et al. 2017). Although our temporally heterogeneous environment contained only a single carbon source at any given point of time, the identity of this carbon source fluctuated randomly over four states every $\sim 13.3$ generations. Therefore, selection was not expected to be blind to the pleiotropic fitness effects of mutations across $\mathrm{T}$, G, M, and S. Despite evolving in such a heterogeneous environment, the FS populations paid significant fitness costs. Thus, Fig. 2 shows that contrary to the expectations of the extant literature (Bono et al. 2017), the presence of multiple dynamic selection pressures can be insufficient for cost avoidance.

Interestingly, evolutionary success in fluctuating environments is reflected by the geometric mean (GM) fitness across the states about which the environment oscillates (and not necessarily the arithmetic mean fitness) (Orr 2007; Kassen 2014). We found that across G, M, S and T, FL had significantly greater GM fitness than both FS and the common ancestor (Fig. S1a; Table 
S5). In contrast, the GM fitness of FS was not significantly different from the ancestral value (Fig. S1a; S5). Furthermore, as expected, evolution in homogeneous (unchanging) environments did not result in increased GM fitness above the ancestral value, regardless of the population size (Fig. S1b and Table S5). FL was better prepared to face the fluctuating environment than all the eight homogeneous environmental regimens (Tables S6 and S8) Surprisingly, the preparedness of FS to face the environmental fluctuations across G, M, S and $\mathrm{T}$ was similar to most homogeneous environment regimens (Tables S7 and S8). These observations highlight the key role played by population size in shaping fitness relationships across the component states of heterogeneous environments. Thus, the mere presence of multiple dynamic selective pressures in a heterogeneous environment was not enough to prevent costs of adaptation, which ultimately precluded any significant increase in the geometric mean fitness of FS.

\section{The genetic basis of cost avoidance}

The observation that FS suffered substantial costs that were completely avoided by FL can be explained by the notion that in the presence of multiple selection pressures, a threshold amount of mutational supply is required to avoid costs. Owing to their relatively larger size, FL are expected to have much higher mutational supply as compared to FS. We hypothesised that FL enriched a larger number of mutations than FS, which made them adapt to multiple carbon sources, thereby avoiding the costs that were paid by the FS populations. To validate this hypothesis, we performed end-point whole-genome whole-population sequencing in three randomly chosen populations each from FL and FS. For our analysis, we considered only mutations that had a frequency $\geq 10 \%$ (Lang et al. 2013; Bailey et al. 2015; Copin et al. 2016; McDonald et al. 2016; Swings et al. 2017). Theory suggests that any mutation rising to frequencies $\geq 10 \%$ within 480 generations in any of our treatment populations is likely to be beneficial and highly unlikely to be neutral or deleterious (Desai \& Fisher 2007a; Good et al. 

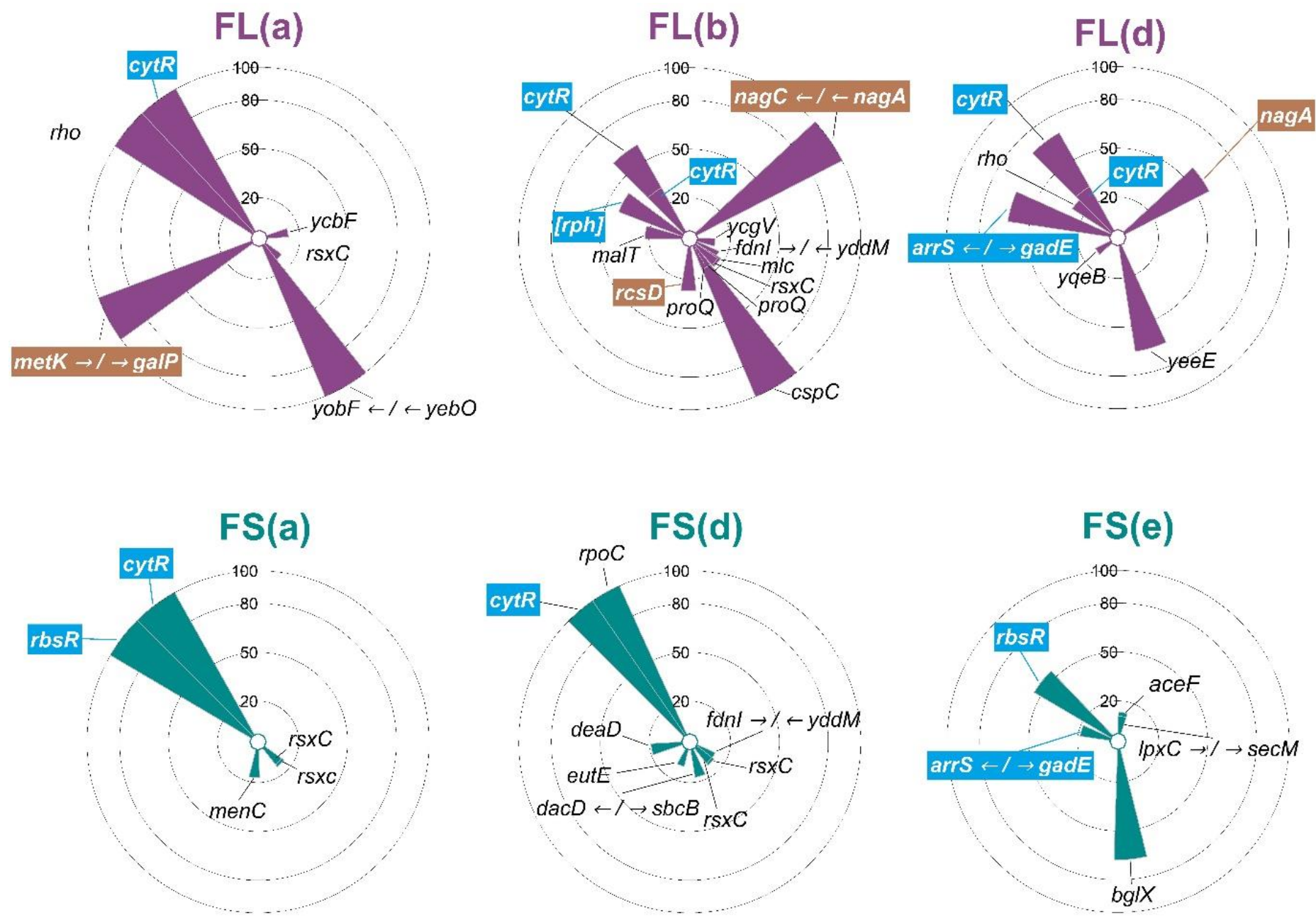

304 Fig. 4. The spectrum of mutations observed in FL and FS after 480 generations. Three randomly chosen replicate populations each of FL (upper row) and FS (lower row) were subjected to whole-genome whole-population sequencing. The radial bars are located at the genomic position of the observed mutations and their heights represent the corresponding mutational frequency. The mutated loci known to be associated with thymidine (T) utilization are highlighted in blue while those associated with galactose $(\mathrm{G})$ utilization are highlighted in brown. See Table S9 for details.

311 A detailed description of the various observed mutations is given in Table S9, while the key observations and their interpretations are described below. 
We found that the loci mutated in FL are known to be associated with the uptake and/or metabolism of either $\mathrm{G}$ or $\mathrm{T}$ in the extant literature. Contrastingly, the loci mutated in FS had known links to T uptake and/or metabolism but none with that of G (Fig. 4). This agrees with the observation that FL adapted to both $\mathrm{T}$ and $\mathrm{G}$ while FS adapted to $\mathrm{T}$ but not to $\mathrm{G}$.

Interestingly, some mutated loci that could be linked with $\mathrm{T}$ adaptation were common across FL and FS while others were found exclusively in either FL or FS. Remarkably, five out of the six sequenced populations (3/3 in FL and $2 / 3$ in FS) had high frequencies of mutations in $c y t R$ (Fig. 4), an important regulator of thymidine metabolism that is instrumental in the regulation of pyrimidine uptake and degradation (Hammer-Jespersen \& Munch-Petersen 1975; ValentinHansen et al. 1996). Similarly, insertions in the upstream regulator region of gadE, a transcriptional activator that plays an important role in thymidine metabolism (Ketcham 2019), were found to be enriched in one replicate of both FL and FS.

A deletion in the expressed but non-active exoribonuclease $r p h$ got enriched in an FL population, but was not found in any of the FS populations (Fig. 4). Such a deletions is likely to affect the expression of $p y r E$, a key gene in thymidine biosynthesis whose promoter lies within rph (Gama-Castro et al. 2016; Wytock et al. 2018). On the other hand, two out of the three sequenced FS populations showed mutations in $r b s R$, the ribose operon repressor that has known links to thymidine metabolism (Shimada et al. 2013). Interestingly, mutations in $r b s R$ did not get enriched in any of the three FL populations.

The mutations identified to be associated with adaptation in Gal were found exclusively in FL (none in FS) and were distributed across diverse loci. For example, mutations influencing the expression of $n a g A$ and $n a g C$ genes were found at frequencies $>50 \%$ in two out of the three sequenced FL populations (Fig. 4). Mutations in these genes are known to increase fitness in galactose minimal media (Soupene et al. 2003; El Qaidi et al. 2009). Similarly, a mutation in 
the operator of galP, the galactose: $\mathrm{H}^{+}$symporter (a gene that is instrumental in galactose uptake) got fixed in one FL population (Fig. 4).

We also found a high frequency mutation directly associated with maltose utilization in one of the FL populations, but none in FS (Fig. 4, Table S9). The presence of this mutation could explain the avoidance of costs in M that could have arisen due to T-associated mutations in FL. Furthermore, we also found several mutations in genes with widespread effects that were not specific to the uptake or metabolism of the carbon sources used in this study (Fig. 4, Table S9).

Since fitness in $\mathrm{T}$ has been shown to be negatively correlated with fitness in G (Chavhan et al. 2020), mutations beneficial in $T$ are likely to be deleterious in $G$, and vice versa. Hence, the presence of several T-associated mutations at high frequencies in FS can explain their maladaptation to G. Moreover, we did not find any known G-associated mutations in FS that could alleviate the putative maladaptive effects of T-associated mutations in G. Had there been no G-associated mutations in FL, the enrichment of a relatively larger number of T-associated mutations should have led to greater maladaptation of FL in G. However, we found several Gassociated mutations at high frequencies in FL that can explain these populations' adaptation to $\mathrm{G}$.

The presence of both T- and G-associated mutations in FL agrees with the observation that this regimen adapted to both $\mathrm{T}$ and $\mathrm{G}$. The large population size of FL could have allowed them to stumble upon highly rare mutations that were simultaneously beneficial in multiple environments ( $\mathrm{Li}$ et al. 2019) ( $\mathrm{T}$ and $\mathrm{G}$ in this case). However, the convergent enrichment of multiple mutations at the level of loci (e.g., within cytR and upstream of gadE (Fig. 4)) in FL and FS makes such a possibility unlikely. Although the investigation of such individual and epistatic effects of mutations on fitness across different environments is interesting in its own 
right, it is outside the scope of our study, which is primarily targeted towards unravelling the interactive effects of population size and environmental heterogeneity in shaping fitness costs.

Taken together, the genomic changes enriched during evolution in the heterogeneous environment were congruent with the phenotypic observation that the large (FL) avoided all the fitness costs that were suffered by the small (FS) populations. Having discussed the match between our phenotypic and genotypic observations, we now turn to the population genetic drivers that could have shaped evolution in our experiments.

Antagonistic pleiotropy can readily explain the positive relationship between population size and fitness costs observed in homogeneous environments (Rose \& Charlesworth 1980; Cohan et al. 1994; Holt 1996; Cooper \& Lenski 2000; Cooper 2014) (Fig. 3). Since these populations faced only one carbon source throughout the experiment, their evolution was blind to fitness changes in other carbon sources. The pleiotropic disadvantages of beneficial mutations are generally expected to be correlated with their direct effects (Lande 1983; Orr \& Coyne 1992; Otto 2004; Chavhan et al. 2019a). Since the larger asexual populations adapt primarily via beneficial mutations with relatively greater direct effect sizes (Desai et al. 2007; Desai \& Fisher 2007b; Sniegowski \& Gerrish 2010; Chavhan et al. 2019b), adapting to homogeneous environments in larger numbers should lead to heavier costs of adaptation, as observed in our study (Fig. 3) (Chavhan et al. 2020).

When evolved in the heterogeneous (fluctuating) environment, smaller (FS) populations paid significant costs of adaptation across three distinct environmental pairs under consideration, but the larger (FL) populations avoided costs altogether. As described above, FS suffered significantly from T-G trade-offs while FL bypassed them. Interestingly, despite facing both T and $\mathrm{G}$ as the sole sources of carbon for equal number of generations $(\sim 120)$, the T-G trade-off 
of fitness changes across $\mathrm{T}$ and $\mathrm{G}$, we note that despite evolving in homogeneous $\mathrm{G}$ for $\sim 480$ generations, GS could not adapt significantly to this environment. Contrastingly, TS increased their fitness in $\mathrm{T}$ by $>1.5$-fold within the same period (Fig. 3). This shows that the size of our small-population regimens was sufficient to adapt significantly to $\mathrm{T}$ but not to $\mathrm{G}$. Put differently, the scope of adaptation in T was much greater than that in G (Chavhan et al. 2020). This can explain why FS adapted to T but not to G. Analogous to TS, such adaptation of FS to T also led to significant maladaptation in the other three environments.

In contrast to the small populations, the large populations in our study had sufficient supply of mutations to adapt to $\mathrm{G}$ within $\sim 480$ generations (Fig. 3). Curiously, we also found that FS could adapt significantly to $\mathrm{G}$ despite encountering this particular environment intermittently for a total period of $\sim 120$ generations (Fig. 2). This observation was also supported by the genome-wide analysis of the evolutionary changes in this regimen, which revealed substantial enrichment of putative G-associated beneficial mutations (Fig. 4).

An important alternative explanation for cost avoidance in heterogeneous environments involves the divergence of the population in question into multiple subpopulations, each one specialized on a different environmental component (Kassen 2002, 2014). However, our genomic data suggest that this explanation of cost avoidance in unlikely in our study. Specifically, in one of the sequenced FL populations (FL(a)), multiple mutations went to fixation, one of which was in a locus known to be associated with galactose uptake/metabolism and another with that of thymidine (Fig. 4). Hence, the individuals in FL(a) simultaneously carried both putative $\mathrm{G}$ and putative $\mathrm{T}$ adaptations. In the second sequenced FL population (FL(b)), a putative G-associated mutation went to fixation and three putative T-associated mutations rose to the frequencies of $60.7 \%, 41.4 \%$, and $32.3 \%$ respectively (Fig. 4, Table S9). Thus, the probability that an individual in FL(b) carried at least one of the three putative $\mathrm{T}$ 
carried a G- and T-associated mutation was $84.41 \%$. Although the third sequenced FL $30.9 \%$, respectively (Fig. 4, Table S9). Hence, the probability that an individual in this of an FL(d) individual simultaneously carrying both G- and T-associated mutations is $53.05 \%$. Thus, the high likelihoods of simultaneously showing G- and T-associated mutations FL suggests that it is unlikely that this regimen avoided costs by divergent specialization on individual carbon sources within populations.

Overall, these results demonstrate that the phenomenon of cost avoidance in heterogeneous (fluctuating) environments requires the supply of variation to be large enough to make use of multiple dynamic selection pressures.

\section{Implications}

Our observations offer a novel explanation for an important conundrum in evolutionary ecology, namely the rarity of detectable fitness costs in empirical studies. Specifically, we demonstrate a previously unreported interaction of population size and environmental heterogeneity that determines the evolutionary appearance (or avoidance) of fitness costs. These results can potentially explain how evolving populations can escape fitness costs despite substantial antagonistic pleiotropy across environmental states. Our study shows that the simultaneity of two conditions, namely large population size and heterogeneous environment, can avoid all the fitness costs that potentially evolve when these conditions are not present together. Finally, to our knowledge, this is the first experimental study to demonstrate that 
multiple mutations can fix rapidly (within $\sim 480$ generations) in asexual populations evolving in highly dynamic heterogeneous environments, a possibility raised recently (Cvijović et al. phenomenon was observed in both FL and FS populations. This shows that such rapid fixation of multiple mutations in heterogeneous environments can happen in the face of both lenient and harsh population bottlenecks.

The environments of most natural populations of asexual microbes are known to be heterogeneous (Green \& Bohannan 2006; Muscarella et al. 2019). Moreover, such natural asexual populations are also known to have extremely large sizes (Torsvik et al. 2002; Tenaillon et al. 2010). Our results suggest that if the asexual population under consideration has a history of evolving in heterogeneous environments in large numbers, it is expected to have reached its current state after having avoided fitness costs during its past evolution. Therefore, if a sample from such a population is now employed to analyse fitness correlations in a single-generation study, such correlations may not be negative, and costs may not be found. environments and large population sizes $\left(>10^{6}\right.$ in terms of harmonic mean population size) have successfully detected fitness costs (Kassen \& Bell 1998; Cooper \& Lenski 2000; Cooper et al. 2001; Nilsson et al. 2004; Hall \& Colegrave 2008; Presloid et al. 2008; Philippe et al. 2009; Vasilakis et al. 2009; Bedhomme et al. 2012; Ensminger et al. 2012; Kubinak \& Potts 2013; Leiby \& Marx 2014). This agrees with the interplay of population size and environmental heterogeneity revealed by our results, which predicts such a combination of constant environment and large populations to lead to significant costs.

453 Thus, apart from explaining why costs may not be detected in single-generation studies with natural isolates, our observations also explain why costs can still be detected if the artificially 
controlled laboratory conditions remain constant over a few hundred generations in an evolution experiment.

457 Although the environments used in our experimental setup were nutritionally challenging

458 minimal media, the explanation of our observations applies to the general notion of fitness costs across multiple environments in asexual microbial populations. In particular, our results can have important implications for understanding the rampant evolution and spread of antibiotic resistance, which has direct practical values. Mutations that confer resistance to antibiotics have been routinely shown to bear fitness costs in drug-free conditions (Andersson \& Hughes 2010; Vogwill \& MacLean 2015). Interestingly, resistant microbes mostly evolve in a heterogeneous environment that fluctuates randomly across antibiotic-laden and antibioticfree conditions (Baquero et al. 1998). Our results predict that small populations evolving in heterogeneous environments suffer heavy fitness costs while large populations are likely to avoid them altogether (Fig. 2). Thus, even if most antibiotic resistance mutations carry a cost in drug-free conditions, large microbial population sizes stemming from lack of sanitary conditions and proper medical waste-disposal (Cantón et al. 2013) could themselves lead to vigorous spread of cost-free resistance.

\section{Methods}

\section{Experimental evolution}

473 We derived ten different evolutionary regimens from a single colony of E. coli MG1655 by culturing populations at two different sizes in five different environments as described above (see Supplementary Methods (SM.1) for more details regarding the ancestral strain and media compositions). Using the standard batch culture technique, we let all the 60 populations 

relatively harsher periodic bottleneck $\left(1: 10^{4}\right.$ dilution $)$. We ensured that populations of different sizes did not remain in the stationary phase for significantly different time-periods by bottlenecking the L populations every $12 \mathrm{hrs}$ ( $\sim 3.3$ generations $)$, and the smaller ones every 48 hrs ( 13.3 generations). The selection protocol pertaining to the $\mathrm{T}$ and $\mathrm{G}$ populations has been reported in a previous study (Chavhan et al. 2020).

We conducted fitness measurements for all the 60 independently evolving populations in all four carbon sources (T, G, M, and S) at the end of the evolution experiment ( 480 generations). To this end, we revived the cryo-stocks belonging to each of the 60 experimental populations in a common nutrient limited environment that was not encountered by any population during the $\sim 480$ generations of our experiment (glucose based M9 minimal medium) and allowed them

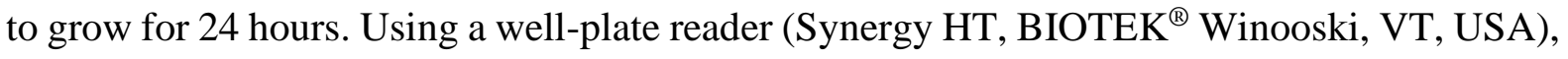
we then performed automated growth measurements on each of the 60 revived populations in all four different minimal media, each based on one of T, G, M, or S. Ensuring that the physical conditions during the fitness measurements were the same as the culture conditions (96 well plates shaken at $150 \mathrm{rpm}$ and ambient temperature maintained at $37^{\circ} \mathrm{C}$ ), we obtained growth readings every 20 minutes for 24 hours. We used optical density (OD) at $600 \mathrm{~nm}$ as the measure of population density.

Since the total number of growth curves was much larger than number of wells in the assay plate, we used a randomized complete block design (RCBD) for growth 
each of the ten different evolutionary lines in all four environments on a given day. Since there were six replicates for each evolutionary line, we conducted growth measurements over six different days. We used the maximum growth rate $(\mathrm{R})$ as the measure of fitness. We computed $\mathrm{R}$ as the maximum slope of the growth curve over a dynamic window of ten OD $(600 \mathrm{~nm})$ readings (Leiby \& Marx 2014; Karve et al. 2015; Chavhan et al. 2019a, b). As described in the Results section, for each of the four sole carbon sources (G, M, S, and T), we used single sample t-tests to compare the fitness of each of the ten evolutionary regimens to that of the ancestor. Subsequently, we corrected for family-wise error rates using the Holm-Šidàk procedure.

As described in the Supplementary Methods, we also investigated the changes in the geometric mean fitness across G, M, S, and T for all the ten evolutionary regimens (see SM.2 for details).

\section{Whole genome whole population sequencing}

For both the ancestor and the six randomly chosen evolved populations (three each from FL and FS), pellets obtained from overnight grown cultures were sent for sequencing to an external service provider. For each sample, the genomic DNA was isolated using c-TAB and phenolchloroform extraction. This procedure was followed by RNAase A treatment. The quality and quantity of the isolated DNA samples was verified using a NanoDrop ${ }^{\mathrm{TM}}$ spectrophotometer (Thermo Fisher Scientific Inc., MA, USA). The isolated DNA samples were initially subjected to a further check by targeting the bacterial 16s gene using Sanger sequencing. After these checks, 2 x 150 NextSeq500 Shotgun Libraries were prepared from each sample using an Illumina TruSeq ${ }^{\circledR}$ Nano DNA Library Prep Kit (Illumina Inc, CA, USA). The quality of each library was checked using the Agilent 4200 Tape Station (Agilent Technologies, CA, USA). 
and paired-end sequencing. Trimmomatic (v0.38) was used to remove adapter sequences, ambiguous reads (with unknown nucleotides > 5\%) and low-quality sequences (reads with > $10 \%$ quality threshold $<20$ phred score). After trimming, a minimum length of 100 nt was applied. The mean coverage across the sequenced populations was $\sim 100$-fold at a quality score of 20 .

We subjected these trimmed high quality sequences to the BRESEQ pipeline (Deatherage \& Barrick 2014) (v0.33.2) to identify mutations enriched during our evolution experiment. We initially compared the ancestral sequence to the reference $E$. coli MG1655 genome to identify differences relative to the latter expected to be found in all the six evolved populations. Next, we adjusted for these differences by using the ancestral sequences as the reference for identifying mutational frequencies in each of the six descendant populations using the 'polymorphic' mode in BRESEQ. To avoid false positives and to restrict our analysis to mutations that must have been instrumental in shaping the average fitness of the population, we ignored mutations with frequencies $<10 \%$.

\section{Acknowledgements}

542 We thank S. Selveshwari for help with NGS analysis and Milind Watve and M.S.

543 Madhusudhan for their valuable inputs. YDC was supported by a Senior Research Fellowship

544 initially sponsored by IISER Pune and then by Council for Scientific and Industrial Research

545 (CSIR), Govt. of India. YDC also acknowledges Department of Biotechnology, Govt. of India

546 for postdoctoral funding for this work. SM was supported by an INSPIRE undergraduate

547 fellowship, sponsored by Department of Science and Technology (DST), Govt. of India. This

548 project was supported by an external grant (BT/PR22328/BRB/10/1569/2016) from

549 Department of Biotechnology, Govt. of India, and internal funding from IISER Pune. 


\section{Conflict of interest}

552 The authors declare that they have no conflict of interest.

553

554 Data archiving

555 All the data relevant to this study will be uploaded on the Dryad digital repository upon 556 acceptance.

\section{References}

Abdi, H. (2010). Holm's sequential Bonferroni procedure. Encycl. Res. Des., 1-8.

Agrawal, A.A., Conner, J.K. \& Rasmann, S. (2010). Tradeoffs and negative correlations in evolutionary ecology. Evol. Darwin First, 150, 243-268.

Ågren, J., Oakley, C.G., McKay, J.K., Lovell, J.T. \& Schemske, D.W. (2013). Genetic mapping of adaptation reveals fitness tradeoffs in Arabidopsis thaliana. Proc. Natl. Acad. Sci., 110, 21077-21082.

Anderson, J.T., Lee, C.-R., Rushworth, C.A., Colautti, R.I. \& Mitchell-Olds, T. (2013). Genetic trade-offs and conditional neutrality contribute to local adaptation. Mol. Ecol., 22, 699-708.

Andersson, D.I. \& Hughes, D. (2010). Antibiotic resistance and its cost: is it possible to reverse resistance? Nat. Rev. Microbiol., 8, 260-271.

Bailey, S.F., Rodrigue, N. \& Kassen, R. (2015). The Effect of Selection Environment on the Probability of Parallel Evolution. Mol. Biol. Evol., 32, 1436-1448.

Baquero, F., Negri, M.-C., Morosini, M.-I. \& Blázquez, J. (1998). Antibiotic-Selective Environments. Clin. Infect. Dis., 27, S5-S11. 
Bedhomme, S., Lafforgue, G. \& Elena, S.F. (2012). Multihost Experimental Evolution of a Plant RNA Virus Reveals Local Adaptation and Host-Specific Mutations. Mol. Biol. Evol., 29, 1481-1492.

Bono, L.M., Smith, L.B., Pfennig, D.W. \& Burch, C.L. (2017). The emergence of performance trade-offs during local adaptation: insights from experimental evolution. Mol. Ecol., 26, 1720-1733.

Cantón, R., Horcajada, J.P., Oliver, A., Garbajosa, P.R. \& Vila, J. (2013). Inappropriate use of antibiotics in hospitals: The complex relationship between antibiotic use and antimicrobial resistance. Enfermedades Infecc. Microbiol. Clínica, 31, Supplement 4, $3-11$.

Chavhan, Y., Karve, S. \& Dey, S. (2019a). Adapting in larger numbers can increase the vulnerability of Escherichia coli populations to environmental changes. Evolution, 73, 836-846.

Chavhan, Y., Malusare, S. \& Dey, S. (2020). Larger bacterial populations evolve heavier fitness trade-offs and undergo greater ecological specialization. Heredity, 1-11.

Chavhan, Y.D., Ali, S.I. \& Dey, S. (2019b). Larger Numbers Can Impede Adaptation in Asexual Populations despite Entailing Greater Genetic Variation. Evol. Biol., 46, 113.

Cohan, F.M., King, E.C. \& Zawadzki, P. (1994). Amelioration of the Deleterious Pleiotropic Effects of an Adaptive Mutation in Bacillus Subtilis. Evolution, 48, 81-95.

Cooper, V.S. (2014). The Origins of Specialization: Insights from Bacteria Held 25 Years in Captivity. PLOS Biol., 12, e1001790.

Cooper, V.S. (2018). Experimental Evolution as a High-Throughput Screen for Genetic Adaptations. mSphere, 3, e00121-18. 
Cooper, V.S., Bennett, A.F. \& Lenski, R.E. (2001). Evolution of Thermal Dependence of Growth Rate of Escherichia Coli Populations During 20,000 Generations in a Constant Environment. Evolution, 55, 889-896.

Cooper, V.S. \& Lenski, R.E. (2000). The population genetics of ecological specialization in evolving Escherichia coli populations. Nature, 407, 736-739.

Copin, R., Wang, X., Louie, E., Escuyer, V., Coscolla, M., Gagneux, S., et al. (2016). Within Host Evolution Selects for a Dominant Genotype of Mycobacterium tuberculosis while T Cells Increase Pathogen Genetic Diversity. PLOS Pathog., 12, e1006111.

Coustau, C., Chevillon, C. \& ffrench-Constant, R. (2000). Resistance to xenobiotics and parasites: can we count the cost? Trends Ecol. Evol., 15, 378-383.

Cvijović, I., Good, B.H., Jerison, E.R. \& Desai, M.M. (2015). Fate of a mutation in a fluctuating environment. Proc. Natl. Acad. Sci., 112, E5021-E5028.

Deatherage, D.E. \& Barrick, J.E. (2014). Identification of Mutations in Laboratory-Evolved Microbes from Next-Generation Sequencing Data Using < Emphasis Type="Italic">breseq</Emphasis>. In: Engineering and Analyzing Multicellular Systems. Humana Press, New York, NY, pp. 165-188.

Desai, M.M. \& Fisher, D.S. (2007a). Beneficial Mutation-Selection Balance and the Effect of Linkage on Positive Selection. Genetics, 176, 1759-1798.

Desai, M.M. \& Fisher, D.S. (2007b). Beneficial mutation-selection balance and the effect of linkage on positive selection. Genetics, 176, 1759-1798.

Desai, M.M., Fisher, D.S. \& Murray, A.W. (2007). The Speed of Evolution and Maintenance of Variation in Asexual Populations. Curr. Biol., 17, 385-394.

El Qaidi, S., Allemand, F., Oberto, J. \& Plumbridge, J. (2009). Repression of galP, the galactose transporter in Escherichia coli, requires the specific regulator of $\mathrm{N}$ acetylglucosamine metabolism. Mol. Microbiol., 71, 146-157. 
Ensminger, A.W., Yassin, Y., Miron, A. \& Isberg, R.R. (2012). Experimental Evolution of Legionella pneumophila in Mouse Macrophages Leads to Strains with Altered Determinants of Environmental Survival. PLOS Pathog., 8, e1002731.

Fisher, R.A. (1930). The Genetical Theory Of Natural Selection. At The Clarendon Press.

Friman, V.-P. \& Buckling, A. (2013). Effects of predation on real-time host-parasite coevolutionary dynamics. Ecol. Lett., 16, 39-46.

Fry, J.D. (1996). The Evolution of Host Specialization: Are Trade-Offs Overrated? Am. Nat., 148, S84-S107.

Futuyma, D.J. \& Moreno, G. (1988). The Evolution of Ecological Specialization. Annu. Rev. Ecol. Syst., 19, 207-233.

Gama-Castro, S., Salgado, H., Santos-Zavaleta, A., Ledezma-Tejeida, D., Muñiz-Rascado, L., García-Sotelo, J.S., et al. (2016). RegulonDB version 9.0: high-level integration of gene regulation, coexpression, motif clustering and beyond. Nucleic Acids Res., 44, D133-D143.

Good, B.H., Rouzine, I.M., Balick, D.J., Hallatschek, O. \& Desai, M.M. (2012). Distribution of fixed beneficial mutations and the rate of adaptation in asexual populations. Proc. Natl. Acad. Sci. U. S. A., 109, 4950-4955.

Green, J. \& Bohannan, B.J.M. (2006). Spatial scaling of microbial biodiversity. Trends Ecol. Evol., 21, 501-507.

Hall, A.R. \& Colegrave, N. (2008). Decay of unused characters by selection and drift. $J$. Evol. Biol., 21, 610-617.

Hammer-Jespersen, K. \& Munch-Petersen, A. (1975). Multiple regulation of nucleoside catabolizing enzymes: Regulation of the $<$ Emphasis Type="Italic" $>$ deo $</$ Emphasis $>$ operon by the $<$ Emphasis Type="Italic" $>$ cytR $</$ Emphasis $>$ and $<$ Emphasis 
Type="Italic">deoR</Emphasis > gene products. Mol. Gen. Genet. MGG, 137, 327335.

Holt, R.D. (1996). Demographic constraints in evolution: Towards unifying the evolutionary theories of senescence and niche conservatism. Evol. Ecol., 10, 1-11.

Jasmin, J.-N. \& Kassen, R. (2007a). Evolution of a single niche specialist in variable environments. Proc. R. Soc. Lond. B Biol. Sci., 274, 2761-2767.

Jasmin, J.-N. \& Kassen, R. (2007b). On the experimental evolution of specialization and diversity in heterogeneous environments. Ecol. Lett., 10, 272-281.

Jasmin, J.-N. \& Zeyl, C. (2013). Evolution of pleiotropic costs in experimental populations. J. Evol. Biol., 26, 1363-1369.

Joshi, A. \& Thompson, J.N. (1995). Trade-offs and the evolution of host specialization. Evol. Ecol., 9, 82-92.

Karve, S.M., Daniel, S., Chavhan, Y.D., Anand, A., Kharola, S.S. \& Dey, S. (2015). Escherichia coli populations in unpredictably fluctuating environments evolve to face novel stresses through enhanced efflux activity. J. Evol. Biol., 28, 1131-1143.

Kassen, R. (2002). The experimental evolution of specialists, generalists, and the maintenance of diversity. J. Evol. Biol., 15, 173-190.

Kassen, R. (2014). Experimental evolution and the nature of biodiversity. Roberts.

Kassen, R. \& Bell, G. (1998). Experimental evolution in Chlamydomonas. IV. Selection in environments that vary through time at different scales. Heredity, 80, 732-741.

Ketcham, A. (2019). Exploring Thymineless Death Using Systems Biology and Laboratory Evolution.

Ketola, T. \& Saarinen, K. (2015). Experimental evolution in fluctuating environments: tolerance measurements at constant temperatures incorrectly predict the ability to tolerate fluctuating temperatures. J. Evol. Biol., 28, 800-806. 
671 Kubinak, J.L. \& Potts, W.K. (2013). Host resistance influences patterns of experimental viral adaptation and virulence evolution. Virulence, 4, 410-418.

Lande, R. (1983). The response to selection on major and minor mutations affecting a metrical trait. Heredity, 50, 47-65.

Lang, G.I., Rice, D.P., Hickman, M.J., Sodergren, E., Weinstock, G.M., Botstein, D., et al. (2013). Pervasive genetic hitchhiking and clonal interference in forty evolving yeast populations. Nature, 500, 571-574.

Leiby, N. \& Marx, C.J. (2014). Metabolic Erosion Primarily Through Mutation Accumulation, and Not Tradeoffs, Drives Limited Evolution of Substrate Specificity in Escherichia coli. PLoS Biol, 12, e1001789.

Levins, R. (1968). Evolution in changing environments: some theoretical explorations. Princeton University Press. Reveals Pareto Fronts that Constrain Adaptation. Nat. Ecol. Evol., 3, 1539-1551.

McDonald, M.J., Rice, D.P. \& Desai, M.M. (2016). Sex speeds adaptation by altering the dynamics of molecular evolution. Nature, 531, 233-236.

Milliken, G.A. \& Johnson, D.E. (2009). Analysis of messy data, volume I: Designed Experiments. Chapman and Hall/CRC.

Muscarella, M.E., Boot, C.M., Broeckling, C.D. \& Lennon, J.T. (2019). Resource heterogeneity structures aquatic bacterial communities. ISME J., 1.

Nilsson, A.I., Kugelberg, E., Berg, O.G. \& Andersson, D.I. (2004). Experimental Adaptation 692 of Salmonella typhimurium to Mice. Genetics, 168, 1119-1130. 
Otto. (2004). Two steps forward, one step back: the pleiotropic effects of favoured alleles.

$$
\text { Proc. R. Soc. Lond. B Biol. Sci., 271, 705-714. }
$$

Philippe, N., Pelosi, L., Lenski, R.E. \& Schneider, D. (2009). Evolution of Penicillin-Binding Protein 2 Concentration and Cell Shape during a Long-Term Experiment with Escherichia coli. J. Bacteriol., 191, 909-921.

Presloid, J.B., Ebendick-Corpus, B.E., Zárate, S. \& Novella, I.S. (2008). Antagonistic Pleiotropy Involving Promoter Sequences in a Virus. J. Mol. Biol., 382, 342-352.

Rausher, M.D. (1984). Tradeoffs in Performance on Different Hosts: Evidence from Withinand Between-Site Variation in the Beetle Deloyala Guttata. Evolution, 38, 582-595.

Remold, S. (2012). Understanding specialism when the jack of all trades can be the master of all. Proc. R. Soc. Lond. B Biol. Sci., rspb20121990.

Rose, M. \& Charlesworth, B. (1980). A test of evolutionary theories of senescence. Nature, 287, 141-142.

Sane, M., Miranda, J.J. \& Agashe, D. (2018). Antagonistic pleiotropy for carbon use is rare in new mutations. Evolution, 72, 2202-2213.

Satterwhite, R.S. \& Cooper, T.F. (2015). Constraints on adaptation of Escherichia coli to mixed-resource environments increase over time. Evolution, 69, 2067-2078.

Schick, A., Bailey, S.F. \& Kassen, R. (2015). Evolution of Fitness Trade-Offs in Locally Adapted Populations of Pseudomonas fluorescens. Am. Nat., 186, S48-S59.

Shimada, T., Kori, A. \& Ishihama, A. (2013). Involvement of the ribose operon repressor RbsR in regulation of purine nucleotide synthesis in Escherichia coli. FEMS Microbiol. Lett., 344, 159-165.

Sniegowski, P.D. \& Gerrish, P.J. (2010). Beneficial mutations and the dynamics of adaptation in asexual populations. Philos. Trans. R. Soc. B Biol. Sci., 365, 1255-1263. 
Soupene, E., Heeswijk, W.C. van, Plumbridge, J., Stewart, V., Bertenthal, D., Lee, H., et al. (2003). Physiological Studies of Escherichia coli Strain MG1655: Growth Defects and Apparent Cross-Regulation of Gene Expression. J. Bacteriol., 185, 5611-5626.

Swings, T., Van, B. den B., Wuyts, S., Oeyen, E., Voordeckers, K., Verstrepen, K.J., et al. (2017). Adaptive tuning of mutation rates allows fast response to lethal stress in Escherichia coli., Adaptive tuning of mutation rates allows fast response to lethal stress in Escherichia coli. ELife ELife, 6, 6.

Tenaillon, O., Skurnik, D., Picard, B. \& Denamur, E. (2010). The population genetics of commensal Escherichia coli. Nat. Rev. Microbiol., 8, 207-217.

Torsvik, V., Øvreås, L. \& Thingstad, T.F. (2002). Prokaryotic Diversity--Magnitude, Dynamics, and Controlling Factors. Science, 296, 1064-1066.

Valentin-Hansen, P., Søgaard-Andersen, L. \& Pedersen, H. (1996). A flexible partnership: the CytR anti-activator and the cAMP-CRP activator protein, comrades in transcription control. Mol. Microbiol., 20, 461-466.

Vasilakis, N., Deardorff, E.R., Kenney, J.L., Rossi, S.L., Hanley, K.A. \& Weaver, S.C. (2009). Mosquitoes Put the Brake on Arbovirus Evolution: Experimental Evolution Reveals Slower Mutation Accumulation in Mosquito Than Vertebrate Cells. PLOS Pathog., 5, e1000467.

Velicer, G.J. \& Lenski, R.E. (1999). Evolutionary Trade-Offs Under Conditions of Resource Abundance and Scarcity: Experiments with Bacteria. Ecology, 80, 1168-1179.

Vila-Aiub, M.M., Neve, P. \& Powles, S.B. (2009). Fitness costs associated with evolved herbicide resistance alleles in plants. New Phytol., 184, 751-767.

Vogwill, T. \& MacLean, R.C. (2015). The genetic basis of the fitness costs of antimicrobial resistance: a meta-analysis approach. Evol. Appl., 8, 284-295. 
744 Wallenstein, M.D. \& Hall, E.K. (2012). A trait-based framework for predicting when and

745

746

747 where microbial adaptation to climate change will affect ecosystem functioning. Biogeochemistry, 109, 35-47.

Whitlock, M.C. (1996). The Red Queen Beats the Jack-Of-All-Trades: The Limitations on the Evolution of Phenotypic Plasticity and Niche Breadth. Am. Nat., 148, S65-S77.

Wytock, T.P., Fiebig, A., Willett, J.W., Herrou, J., Fergin, A., Motter, A.E., et al. (2018). Experimental evolution of diverse Escherichia coli metabolic mutants identifies genetic loci for convergent adaptation of growth rate. PLoS Genet., 14. 
754 An interplay of population size and environmental heterogeneity explains why fitness costs are rare

756

757

Yashraj Chavhan ${ }^{1}$, Sarthak Malusare $^{1}$, and Sutirth Dey ${ }^{1, *}$

${ }^{1}$ Indian Institute of Science Education and Research (IISER) Pune, Dr Homi Bhabha Road, 759 Pashan, Pune, Maharashtra, 411008, India.

$760 *$ Corresponding author: Sutirth Dey, Biology Division, Indian Institute of Science-Education and Research Pune, Dr Homi Bhabha Road, Pune, Maharashtra 411 008, India. 
Table S1. The absence of costs in experimental evolution studies with asexual microbes

\begin{tabular}{|c|c|c|}
\hline Reference no. & Model system & Absence of costs \\
\hline 1 & Bacteriophage $\varphi 6$ & uniform \\
\hline 2 & Bacteriophage $\varphi 6$ & nonuniform \\
\hline 3 & Bacteriophage $\varphi 6$ & nonuniform \\
\hline 4 & Bacteriophage $\varphi 6$ & nonuniform \\
\hline 5 & Bacteriophage $\varphi 6$ & nonuniform \\
\hline 6 & Bacteriophage ID8 and NC28 & uniform \\
\hline 7 & Burkholderia sp. & nonuniform \\
\hline 8 & Chlamydomonas reinhardtii & uniform \\
\hline 9 & Cucumber mosaic virus & uniform \\
\hline 10 & Dengue virus & uniform \\
\hline 11 & Escherichia coli & uniform \\
\hline 12 & Escherichia coli & nonuniform \\
\hline 13 & Escherichia coli & nonuniform \\
\hline 14 & Escherichia coli & nonuniform \\
\hline 15 & Escherichia coli & nonuniform \\
\hline 16 & Escherichia coli & nonuniform \\
\hline 17 & Escherichia coli & nonuniform \\
\hline 18 & Holospora undulata & nonuniform \\
\hline 19 & Pseudomonas aeruginosa & nonuniform \\
\hline 20 & Pseudomonas fluorescens & uniform \\
\hline 21 & Pseudomonas fluorescens & uniform \\
\hline 22 & Pseudomonas fluorescens & nonuniform \\
\hline 23 & Pseudomonas fluorescens & nonuniform \\
\hline 24 & Pseudomonas fluorescens & nonuniform \\
\hline 25 & Saccharomyces cerevisiae & nonuniform \\
\hline 26 & Saccharomyces cerevisiae & nonuniform \\
\hline 27 & Saccharomyces cerevisiae & nonuniform \\
\hline 28 & Saccharomyces cerevisiae & nonuniform \\
\hline 29 & Serratia marcescens & nonuniform \\
\hline
\end{tabular}



experimental populations

\section{ST.1. Details of the studies shown in Fig. 1a:}

764 Fig. 1a incorporates those bacterial experimental evolution studies on fitness costs in 765 heterogeneous environments for which estimates of harmonic mean population size could be 766 obtained. Studies conducted with viruses, and eukaryotes are not included here.

767 Key in the legend of Fig. 1a:

768 A: Ref. 29*; B: Ref. 24; C: Ref. 19; D: Ref. 23; E: Ref. 15; F: Ref. 11; G: Ref. 22; H: Ref. 20; 769 I: Ref. $17^{\ddagger}$

770 *The population size reported for Study A (Ref. 29) has been calculated indirectly using the 771 stationary phase densities reported for a different bacterial species in the selection medium in 772 question and is likely an overestimate.

773 The data for population size have been provided by the authors of Study I (Ref. 17). 


\section{Supplementary Methods}

\section{SM.1. Details of the ancestral strain and nutrient media:}

776

777

778

779

Ancestral strain: Escherichia coli MG1655 lacY::kan. The ancestral strain was resistant to kanamycin.

Nutrient media: There was one heterogeneous and four homogeneous environments in our evolution experiment. Each homogeneous environment comprised of an M9-based minimal medium, 1 litre of which contained the following:

- $\quad 12.8 \mathrm{~g} \mathrm{Na}_{2} \mathrm{HPO}_{4} .7 \mathrm{H}_{2} \mathrm{O}$

- $3.0 \mathrm{~g} \mathrm{KH}_{2} \mathrm{PO}_{4}$

- $0.5 \mathrm{~g} \mathrm{NaCl}$

- $1.0 \mathrm{~g} \mathrm{NH}_{4} \mathrm{Cl}$

- $240.6 \mathrm{mg} \mathrm{MgSO}_{4}$

- $11.1 \mathrm{mg} \mathrm{CaCl} 2$

- $4 \mathrm{~g}$ of the pre-decided sole carbon source

- 50 mg Kanamycin sulphate

The four homogeneous environments differed in terms of the identity of the pre-decided sole carbon source. The following four carbon sources were used in our experiment:

- Thymidine

- Galactose

- Maltose

- Sorbitol

The heterogeneous environment fluctuated randomly between the above four carbon sources every 13.3 generations. 
We computed the geometric mean fitness across each of the four carbon sources (G, M, S, and $\mathrm{T}$ ) for all the ten evolutionary regimens in our experiment (comprising sixty independently evolving populations in total).

We used a mixed model ANOVA to compare the geometric mean fitness across the populations evolved in the heterogeneous environment (FL and FS). In this analysis, we considered the population size (two levels: large (L) and small (S)) as the fixed factor and the day of assay as the random factor, with each day corresponding to one biological replicate in our randomized complete block design (RCBD (see the Main text for details)). We also determined the effect size of the difference between FL and FS using partial $\eta^{2}$, interpreting the latter as showing small, medium, or large effect for Partial $\eta^{2}<0.06,0.06<$ Partial $\eta^{2}<0.14,0.14<$ Partial $\eta^{2}$ respectively ${ }^{31}$.

We further tested if the treatment regimens evolved in the heterogeneous environment (FL / FS) had evolved significantly different geometric mean fitness (over T, G, M, and S) as compared to the control regimens evolved in homogeneous environments. To this end, we conducted two mixed-model ANOVAs with evolutionary regimen (nine levels) as the fixed factor and day of assay (six levels) as the random factor. In the first ANOVA (Table S6), the nine levels in the evolutionary regimen (fixed factor) consisted of the eight homogeneous environments regimens and FL, while in the second ANOVA (Table S7), the fixed factor consisted of the same eight homogeneous environments and FS. For both ANOVAs, we used the Dunnett's procedure) to assess the pairwise differences of FL or FS with the eight homogeneous environment regimens.

In another (more conservative) analysis of the differences in GM fitness across regimens, we used a mixed model ANOVA with evolutionary regimen (ten levels: FL, FS and eight homogeneous environment regimens) as the fixed factor and day of assay (six levels) as the random factor. Subsequently, we compared all possible pairwise differences between the ten evolutionary regimens using Tukey's HSD (Table S8). 


\section{Supplementary Results}

Table S2. Analysis of adaptation and maladaptation events in all ten evolutionary regimens using single-sample t-tests $(\mathrm{N}=6)$ with reference to the ancestral fitness in each of the four carbon sources* (scaled to 1).

\begin{tabular}{|c|c|c|c|c|c|}
\hline $\begin{array}{l}\text { Selection } \\
\text { environment }\end{array}$ & $\begin{array}{l}\text { Population } \\
\text { type }\end{array}$ & $\begin{array}{l}\text { Assay } \\
\text { environment }\end{array}$ & $P$ value & $\begin{array}{r}\text { Corrected } P \\
\text { value } \\
\end{array}$ & Inference \\
\hline Heterogeneous & FL & $\mathrm{T}$ & $1.58 \times 10^{-5}$ & $6.33 \times 10^{-5}$ & Adaptation \\
\hline Heterogeneous & FL & G & $7.025 \times 10^{-3}$ & 0.021 & Adaptation \\
\hline Heterogeneous & FL & M & 0.564 & - & No change \\
\hline Heterogeneous & FL & S & 0.612 & - & No change \\
\hline Heterogeneous & FS & $\mathrm{T}$ & 0.049 & 0.049 & Adaptation \\
\hline Heterogeneous & FS & G & 0.025 & 0.051 & Maladaptation \\
\hline Heterogeneous & FS & M & $2.5 \times 10^{-4}$ & 0.001 & Maladaptation \\
\hline Heterogeneous & FS & S & 0.009 & 0.02635 & Maladaptation \\
\hline Homogeneous $\mathrm{T}$ & TL & $\mathrm{T}$ & $8.1 \times 10^{-7}$ & $3.24 \times 10^{-6}$ & Adaptation \\
\hline Homogeneous $\mathrm{T}$ & $\mathrm{TL}$ & G & $8.94 \times 10^{-5}$ & $2.68 \times 10^{-4}$ & Maladaptation \\
\hline Homogeneous $\mathrm{T}$ & $\mathrm{TL}$ & M & $5.53 \times 10^{-4}$ & $5.53 \times 10^{-4}$ & Maladaptation \\
\hline Homogeneous T & $\mathrm{TL}$ & S & $1.13 \times 10^{-4}$ & $2.27 \times 10^{-4}$ & Maladaptation \\
\hline Homogeneous $\mathrm{T}$ & TS & $\mathrm{T}$ & $1.83 \times 10^{-4}$ & $7.33 \times 10^{-4}$ & Adaptation \\
\hline Homogeneous $\mathrm{T}$ & $\mathrm{TS}$ & G & $6.839 \times 10^{-3}$ & $6.839 \times 10^{-3}$ & Maladaptation \\
\hline Homogeneous $\mathrm{T}$ & $\mathrm{TS}$ & M & $4.58 \times 10^{-4}$ & $1.372 \times 10^{-3}$ & Maladaptation \\
\hline Homogeneous $\mathrm{T}$ & TS & S & $6.19 \times 10^{-4}$ & $1.238 \times 10^{-3}$ & Maladaptation \\
\hline Homogeneous G & GL & $\mathrm{T}$ & 0.003 & 0.013 & Maladaptation \\
\hline Homogeneous $\mathrm{G}$ & GL & G & 0.016 & 0.049 & Adaptation \\
\hline Homogeneous G & GL & M & 0.633 & 0.633 & No change \\
\hline Homogeneous G & GL & $\mathrm{S}$ & 0.973 & 0.973 & No change \\
\hline Homogeneous G & GS & $\mathrm{T}$ & 0.122 & 0.122 & No change \\
\hline Homogeneous $\mathrm{G}$ & GS & G & 0.617 & 0.617 & No change \\
\hline
\end{tabular}




\begin{tabular}{lllcrr} 
Homogeneous G & GS & M & 0.483 & 0.483 & No change \\
Homogeneous G & GS & S & 0.016 & 0.061 & No change \\
\hline & & & & continued...
\end{tabular}

Table S2 continued

\begin{tabular}{|c|c|c|c|c|c|}
\hline $\begin{array}{l}\text { Selection } \\
\text { environment }\end{array}$ & $\begin{array}{l}\text { Population } \\
\text { type }\end{array}$ & $\begin{array}{l}\text { Assay } \\
\text { environment }\end{array}$ & $P$ value & $\begin{array}{r}\text { Corrected } P \\
\text { value }\end{array}$ & Inference \\
\hline Homogeneous M & ML & $\mathrm{T}$ & 0.252 & - & No change \\
\hline Homogeneous M & ML & G & 0.036 & 0.134661 & No change \\
\hline Homogeneous M & ML & M & 0.090 & - & No change \\
\hline Homogeneous M & ML & S & 0.762 & - & No change \\
\hline Homogeneous M & MS & $\mathrm{T}$ & 0.134 & - & No change \\
\hline Homogeneous M & MS & G & 0.164 & - & No change \\
\hline Homogeneous M & MS & M & 0.066 & - & No change \\
\hline Homogeneous M & MS & $S$ & 0.069 & - & No change \\
\hline Homogeneous $\mathrm{S}$ & SL & $\mathrm{T}$ & $3.14 \times 10^{-4}$ & $1.257 \times 10^{-3}$ & Maladaptation \\
\hline Homogeneous S & SL & $\mathrm{G}$ & $7.59 \times 10^{-3}$ & 0.023 & Maladaptation \\
\hline Homogeneous S & SL & M & 0.088 & - & No change \\
\hline Homogeneous S & SL & $S$ & 0.690 & - & No change \\
\hline Homogeneous $\mathrm{S}$ & SS & $\mathrm{T}$ & $5.5 \times 10^{-3}$ & 0.011 & Maladaptation \\
\hline Homogeneous S & SS & $\mathrm{G}$ & $6.4 \times 10^{-5}$ & $2.56 \times 10^{-4}$ & Maladaptation \\
\hline Homogeneous $\mathrm{S}$ & SS & M & 0.001 & 0.003 & Maladaptation \\
\hline Homogeneous $\mathrm{S}$ & SS & $\mathrm{S}$ & 0.366 & - & No change \\
\hline
\end{tabular}


Table S3. The evolutionary emergence of costs of adaptation in populations evolved in the heterogeneous environment

\begin{tabular}{|c|r|c|c|}
\hline \multicolumn{2}{|c|}{ Case(s) which showed costs of adaptation } & \multicolumn{2}{c|}{ Case(s) which showed simultaneous adaptation } \\
\hline $\begin{array}{c}\text { Population } \\
\text { size }\end{array}$ & Environmental pair(s) & $\begin{array}{c}\text { Population } \\
\text { size }\end{array}$ & Environmental pair(s) \\
\hline Large & None & Large & T (adaptation) - G (adaptation) \\
\hline \multirow{2}{*}{ Small } & $\mathrm{T}$ (adaptation) - G (maladaptation) & & \\
\cline { 2 - 3 } & $\mathrm{T}$ (adaptation) - S (maladaptation) & Small & None \\
\hline & $\mathrm{T}$ (adaptation) - M (maladaptation) & & \\
\hline
\end{tabular}

Table S4. The evolutionary emergence of costs of adaptation in populations evolved in homogeneous environments

\begin{tabular}{|c|c|c|c|}
\hline \multicolumn{3}{|c|}{ Case(s) which showed costs of adaptation } & \multirow{2}{*}{$\begin{array}{c}\text { Case(s) which showed } \\
\text { simultaneous } \\
\text { adaptation }\end{array}$} \\
\hline $\begin{array}{c}\text { Selection } \\
\text { environment }\end{array}$ & $\begin{array}{c}\text { Population } \\
\text { size }\end{array}$ & Environmental pair(s) & \\
\hline \multirow{3}{*}{$\mathrm{T}$} & \multirow{3}{*}{ Large } & $\mathrm{T}$ (adaptation) - G (maladaptation) ${ }^{\dagger}$ & \multirow{10}{*}{ None } \\
\hline & & $\mathrm{T}$ (adaptation) - S (maladaptation) ${ }^{\dagger}$ & \\
\hline & & $\mathrm{T}$ (adaptation) - M (maladaptation) ${ }^{\dagger}$ & \\
\hline \multirow{3}{*}{$\mathrm{T}$} & \multirow{3}{*}{ Small } & $\mathrm{T}$ (adaptation) - G (maladaptation) & \\
\hline & & $\mathrm{T}$ (adaptation) - S (maladaptation) & \\
\hline & & $\mathrm{T}$ (adaptation) - M (maladaptation) & \\
\hline G & Large & G (adaptation) - T (maladaptation) & \\
\hline G & Small & \multirow{3}{*}{$\begin{array}{c}\text { Not applicable } \\
\text { (no adaptation to the selection } \\
\text { environment) }\end{array}$} & \\
\hline$S$ & Large & & \\
\hline$S$ & Small & & \\
\hline
\end{tabular}




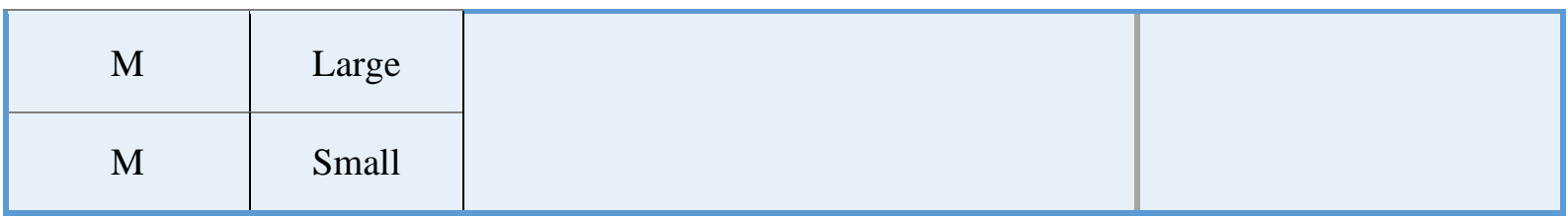

${ }^{\dagger}$ Across the T - M, T - G, and T - S pairs, the costs suffered by the large (TL) populations were greater than those suffered by the small (TS) populations ${ }^{32}$.
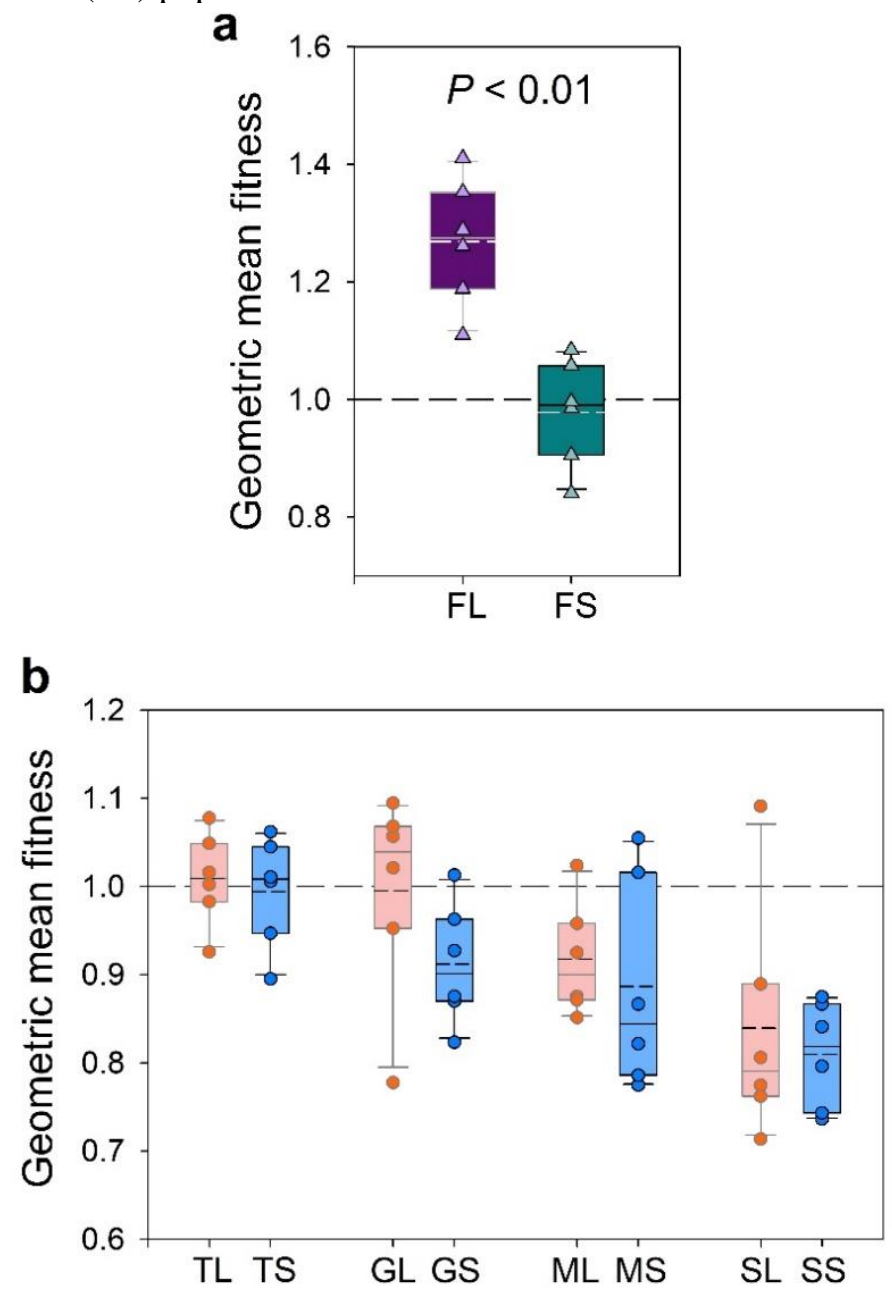
$\mathbf{M}$, and S. The solid lines in the box plots mark the $25^{\text {th }}, 50^{\text {th }}$, and $75^{\text {th }}$ percentiles while the whiskers mark the $10^{\text {th }}$ and $90^{\text {th }}$ percentiles; the short-dashed lines within the box plots represent means $(\mathrm{N}=6)$. The long-dashed line outside the box plots represent the ancestral level of the ordinate. (a) Geometric mean fitness of populations evolved in the heterogeneous environment. FL > FS (P<0.01). (b) Geometric mean fitness of populations evolved in homogeneous environments. See Tables S5 and S6 for details.

833 We found that FL populations had significantly higher geometric mean fitness than FS (Fig. 5a; Table S4; mixed-model ANOVA: $\mathrm{F}_{1,5}=18.002 ; P=0.008$; partial $\eta^{2}=0.783$ (large effect)). Thus, the large (FL) populations adapted better than the small (FS) populations to their 
common heterogeneous environment. This result is expected from the absence of any fitness costs in FL and the presence of such costs across the maximum possible number of environmental pairs under consideration in FS. We further found that FL could significantly enhance their geometric mean fitness with respect to the common ancestor, but FS failed to do 840 so (Fig. S1a; Table S5). Curiously, despite showing significant fitness changes in $\mathrm{T}$ (adaptation) and G (maladaptation), FS did not have significantly different geometric mean fitness as compared to the common ancestor (Fig. S1a; Table S5). Adaptation to homogeneous environments is not expected to entail increased geometric mean fitness over multiple (unencountered) environments. Indeed, we found that the geometric mean fitness over the four carbon sources did not increase significantly as compared to the ancestral level in any of the homogeneous environment regimens, regardless of the population size (Fig. S1b; Table S5).

We also found that FL had a much larger geometric mean fitness than all the homogeneous environment regimens (Table S6). However, FS did not have significantly different geometric mean fitness as compared to a vast majority (seven out of the eight) of homogeneous environment regimens (Table S7). A similar pattern was revealed by a more conservative post hoc analysis using Tukey's HSD (Table S8).

Both the above analyses (using Dunnett's or Tukey's post-hoc tests) sought to answer the same question: whether the FL / FS regimens significantly differed in their GM fitness as compared to the homogeneous environment regimens. Comparing Tables S6-S7 with S8, we find that the pair-wise differences that turn up as statistically significant are identical between the two analyses (except one case: FS and SL show up as significantly different in Dunnett's test but not in Tukey's HSD). This is not surprising, as the analysis with two Dunnett's procedures comprises of (and therefore corrects for) only 18 pair-wise tests, while the corresponding analysis with Tukey's HSD corrects for 81 pair-wise tests (of which only 18 are relevant for our purpose). Therefore, the second analysis has a lot less power than the first one. The fact 
that the results remain virtually identical across both cases highlights the robustness of the same. It should be noted here that our interpretation of the difference between FL and FS remains agnostic to the choice of analysis.

864 Taken together, FL adapted significantly to the heterogeneous (fluctuating) environment, but

Table S5. Summary of single-sample t-tests $(\mathrm{N}=6)$ of differences in the geometric mean fitness (calculated over $\mathrm{G}$, $\mathrm{M}, \mathrm{S}$, and $\mathrm{T}$ ) of the ten evolutionary regimens with the corresponding ancestral value $(=1)$

\begin{tabular}{llrl}
\hline $\begin{array}{l}\text { Selection } \\
\text { environment }\end{array}$ & Population type & $P$ value & Inference \\
\hline Heterogeneous & FL & 0.002 & GM enhanced \\
Heterogeneous & FS & 0.584 & No change \\
Homogeneous & TL & 0.703 & No change \\
Homogeneous & TS & 0.826 & No change \\
Homogeneous & GL & 0.922 & No change \\
Homogeneous & GS & 0.026 & GM reduced \\
Homogeneous & ML & 0.027 & GM reduced \\
Homogeneous & MS & 0.069 & No change \\
Homogeneous & SL & 0.034 & GM reduced \\
Homogeneous & SS & $5.96 \times 10^{-4}$ & GM reduced \\
\hline
\end{tabular}

Table S6. Summary of Dunnett post-hoc tests $(\mathrm{N}=6)$ with respect to FL done after analysing the geometric mean fitness differences across nine evolutionary regimens (FL and eight homogeneous environment regimens) using a mixed model ANOVA, which revealed a significant main effect of the identity of the evolutionary regimen: $\mathrm{F}_{8,40}=16.284, P=2.172 \times 10^{-10}$

\begin{tabular}{lr}
\hline Population type & $P$ value (Dunnett (reference: FL)) \\
\hline GL & 0.000016 \\
GS & 0.000009 \\
TL & 0.000028 \\
TS & 0.000016 \\
ML & 0.000009
\end{tabular}


Table S7. Summary of Dunnett post-hoc tests $(\mathrm{N}=6)$ with respect to FS done after analysing the geometric mean fitness differences across nine evolutionary regimens (FS and eight homogeneous environment regimens) using a mixed model ANOVA, which revealed a significant main effect of the identity of the evolutionary regimen: $\mathrm{F}_{8,40}=5.094, P=2.074 \times 10^{-4}$

\begin{tabular}{lr}
\hline Population type & $P$ value (Dunnett (reference: FS)) \\
\hline GL & 0.999771 \\
GS & 0.577267 \\
TL & 0.984290 \\
TS & 0.999855 \\
ML & 0.666052 \\
MS & 0.243947 \\
SL & 0.024777 \\
SS & 0.004229 \\
\hline
\end{tabular}

873

874

875

Table S8. Summary of Tukey post-hoc tests $(\mathrm{N}=6)$ done after analysing the geometric mean fitness differences across all the ten evolutionary regimens using a mixed model ANOVA, which revealed a significant main effect of the identity of the evolutionary regimen: $\mathrm{F}_{9,45}=14.566, P=1.129 \times 10^{-10}$. Tukey $P$ values for pairwise differences with only FL and FS are shown below:

\begin{tabular}{l|l|l|l|l|l|l|l|l|l|l|}
\multicolumn{2}{l}{} & GL & GS & TL & TS & ML & MS & SL & SS & FL \\
FL & 0.000177 & 0.000156 & 0.000216 & 0.000175 & 0.000156 & 0.000156 & 0.000156 & 0.000156 & - & 0.000161 \\
\cline { 2 - 12 } FS & 0.999998 & 0.921270 & 0.999688 & 0.999999 & 0.952902 & 0.647098 & 0.126786 & 0.027274 & 0.000161 & - \\
\hline
\end{tabular}


Table S9. Details of mutations observed at frequencies $\geq 10 \%$ after $\sim 480$ generations of evolution

\begin{tabular}{|c|c|c|c|c|c|c|c|}
\hline Population & Position & Mutation & Frequency & Annotation & Locus/Region & Region description & Putative link to adaptation \\
\hline $\mathrm{FL}(\mathrm{A})$ & $19,09,523$ & IS2 $(+)+5$ bp & $100 \%$ & intergenic $(-22 /+644)$ & yobF $\leftarrow / \leftarrow$ yebO & $\begin{array}{l}\text { DUF2527 domain-containing protein } \\
\text { YobF / uncharacterized protein YebO }\end{array}$ & - \\
\hline $\mathrm{FL}(\mathrm{A})$ & $30,89,333$ & $\mathrm{C} \rightarrow \mathrm{A}$ & $100 \%$ & intergenic $(+341 /-83)$ & metK $\rightarrow / \rightarrow$ galP & $\begin{array}{l}\text { methionine adenosyltransferase / } \\
\text { galactose: } \mathrm{H}(+) \text { symporter }\end{array}$ & Galactose uptake \\
\hline FL(A) & $39,67,800$ & $\mathrm{G} \rightarrow \mathrm{T}$ & $100 \%$ & S84I (AGC $\rightarrow$ ATC) & rho $\rightarrow$ & transcription termination factor Rho & - \\
\hline $\mathrm{FL}(\mathrm{A})$ & $41,25,478$ & $\mathrm{G} \rightarrow \mathrm{A}$ & $100 \%$ & $\mathrm{Q} 38 *(\mathrm{CAG} \rightarrow \mathrm{TAG})$ & cytR $\leftarrow$ & $\begin{array}{l}\text { DNA-binding transcriptional repressor } \\
\text { CytR }\end{array}$ & Thymidine metabolism \\
\hline $\mathrm{FL}(\mathrm{A})$ & $10,04,716$ & $\mathrm{~A} \rightarrow \mathrm{C}$ & $12.80 \%$ & T66P $(A C G \rightarrow C C G)$ & $\mathrm{ycbF} \rightarrow$ & putative fimbrial chaperone $\mathrm{YcbF}$ & - \\
\hline $\mathrm{FL}(\mathrm{A})$ & $17,10,904$ & $\mathrm{~A} \rightarrow \mathrm{C}$ & $11.60 \%$ & $\mathrm{~A} 692 \mathrm{~A}(\mathrm{GCA} \rightarrow \mathrm{GCC})$ & $\mathrm{rsxC} \rightarrow$ & $\begin{array}{l}\text { SoxR }[2 \mathrm{Fe}-2 \mathrm{~S}] \text { reducing system protein } \\
\text { RsxC }\end{array}$ & - \\
\hline FL(B) & $7,02,169$ & $\mathrm{~A} \rightarrow \mathrm{C}$ & $100 \%$ & intergenic $(-1 /+8)$ & $\operatorname{nagC} \leftarrow / \leftarrow \operatorname{nag} \mathrm{A}$ & $\begin{array}{l}\text { DNA-binding transcriptional dual } \\
\text { regulator NagC / } \\
\text { N-acetylglucosamine-6-phosphate } \\
\text { deacetylase }\end{array}$ & Galactose metabolism \\
\hline $\mathrm{FL}(\mathrm{B})$ & $19,09,302$ & $\operatorname{IS5}(-)+4 \mathrm{bp}$ & $100 \%$ & coding $(41-44 / 210 \mathrm{nt})$ & $\operatorname{cspC} \leftarrow$ & $\begin{array}{l}\text { stress protein, member of the CspA } \\
\text { family }\end{array}$ & - \\
\hline $\mathrm{FL}(\mathrm{B})$ & $41,24,957$ & $\Delta 8 \mathrm{bp}$ & $60.70 \%$ & coding (626-633/1026 nt) & cytR $\leftarrow$ & $\begin{array}{l}\text { DNA-binding transcriptional repressor } \\
\text { CytR }\end{array}$ & Thymidine metabolism \\
\hline $\mathrm{FL}(\mathrm{B})$ & $38,16,992$ & $\Delta 82 \mathrm{bp}$ & $41.40 \%$ & & [rph] & [rph] & Thymidine metabolism \\
\hline $\mathrm{FL}(\mathrm{B})$ & $41,25,100$ & $\Delta 1 \mathrm{bp}$ & $32.30 \%$ & coding $(490 / 1026 n t)$ & cytR $\leftarrow$ & $\begin{array}{l}\text { DNA-binding transcriptional repressor } \\
\text { CytR }\end{array}$ & Thymidine metabolism \\
\hline $\mathrm{FL}(\mathrm{B})$ & $23,17,127$ & $\mathrm{IS} 2(-)+5 \mathrm{bp}$ & $27.00 \%$ & coding $(2508-2512 / 2673 \mathrm{nt})$ & $\mathrm{rcsD} \rightarrow$ & RcsD phosphotransferase & Galactose metabolism \\
\hline $\mathrm{FL}(\mathrm{B})$ & $35,54,248$ & $\mathrm{~T} \rightarrow \mathrm{A}$ & $22.20 \%$ & V11D $($ GTT $\rightarrow$ GAT $)$ & malT $\rightarrow$ & $\begin{array}{l}\text { DNA-binding transcriptional activator } \\
\text { MalT }\end{array}$ & Maltose metabolism \\
\hline $\mathrm{FL}(\mathrm{B})$ & $16,70,246$ & IS2 $(-)+5 \mathrm{bp}$ & $17.40 \%$ & coding $(225-229 / 1221 \mathrm{nt})$ & $\mathrm{mlc} \leftarrow$ & $\begin{array}{l}\text { DNA-binding transcriptional repressor } \\
\text { Mlc }\end{array}$ & - \\
\hline $\mathrm{FL}(\mathrm{B})$ & $17,10,904$ & $\mathrm{~A} \rightarrow \mathrm{C}$ & $16.20 \%$ & $\mathrm{~A} 692 \mathrm{~A}(\mathrm{GCA} \rightarrow \mathrm{GCC})$ & $\mathrm{rsxC} \rightarrow$ & $\begin{array}{l}\text { SoxR }[2 \mathrm{Fe}-2 \mathrm{~S}] \text { reducing system protein } \\
\text { RsxC }\end{array}$ & - \\
\hline $\mathrm{FL}(\mathrm{B})$ & $19,17,415$ & $\Delta 1 \mathrm{bp}$ & $15.40 \%$ & coding $(30 / 699 \mathrm{nt})$ & proQ $\leftarrow$ & RNA chaperone ProQ & - \\
\hline FL(B) & $19,17,411$ & +TTTACTGCTAT & $14.80 \%$ & coding (34/699 nt) & proQ $\leftarrow$ & RNA chaperone ProQ & - \\
\hline
\end{tabular}


Table S9 (continued)

\begin{tabular}{|c|c|c|c|c|c|c|c|}
\hline Population & Position & Mutation & Frequency & Annotation & Locus/Region & Region description & Putative link to adaptation \\
\hline $\mathrm{FL}(\mathrm{B})$ & $15,54,101$ & $\mathrm{C} \rightarrow \mathrm{A}$ & $14.40 \%$ & intergenic $(+200 /+207)$ & $\mathrm{fdnI} \rightarrow / \leftarrow$ yddM & $\begin{array}{l}\text { formate dehydrogenase } \mathrm{N} \text { subunit } \\
\text { gamma / putative DNA-binding } \\
\text { transcriptional regulator YddM }\end{array}$ & - \\
\hline $\mathrm{FL}(\mathrm{B})$ & $12,55,907$ & $\mathrm{~T} \rightarrow \mathrm{G}$ & $10.60 \%$ & $\mathrm{~K} 652 \mathrm{~N}(\mathrm{AAA} \rightarrow \mathrm{AAC})$ & $\mathrm{ycgV} \leftarrow$ & putative autotransporter adhesin $\mathrm{YcgV}$ & - \\
\hline FL(D) & $41,24,871$ & $\mathrm{~A} \rightarrow \mathrm{G}$ & $68.50 \%$ & $\mathrm{~L} 240 \mathrm{P}(\mathrm{CTT} \rightarrow \mathrm{CCT})$ & cytR $\leftarrow$ & $\begin{array}{l}\text { DNA-binding transcriptional repressor } \\
\text { CytR }\end{array}$ & Thymidine metabolism \\
\hline FL(D) & $20,86,018$ & $\mathrm{G} \rightarrow \mathrm{A}$ & $65.50 \%$ & $\mathrm{~A} 214 \mathrm{~A}(\mathrm{GCC} \rightarrow \mathrm{GCT})$ & yeeE $\leftarrow$ & inner membrane protein YeeE & - \\
\hline FL(D) & $36,59,405$ & IS5 (-) +4 bp & $63.00 \%$ & intergenic (-218/-91) & $\operatorname{arrS} \leftarrow / \rightarrow \operatorname{gadE}$ & $\begin{array}{l}\text { small regulatory RNA } \\
\text { ArrS/DNA-binding transcriptional } \\
\text { activator GadE }\end{array}$ & Thymidine metabolism \\
\hline FL(D) & $7,02,933$ & $\mathrm{~T} \rightarrow \mathrm{G}$ & $57.70 \%$ & E131D $($ GAA $\rightarrow$ GAC) & $\operatorname{nagA} \leftarrow$ & $\begin{array}{l}\mathrm{N} \text {-acetylglucosamine-6-phosphate } \\
\text { deacetylase }\end{array}$ & Galactose metabolism \\
\hline FL(D) & $41,24,679$ & $\mathrm{C} \rightarrow \mathrm{T}$ & $30.90 \%$ & G304D (GGT $\rightarrow$ GAT) & cytR $\leftarrow$ & $\begin{array}{l}\text { DNA-binding transcriptional repressor } \\
\text { CytR }\end{array}$ & Thymidine metabolism \\
\hline FL(D) & $39,67,875$ & $\mathrm{G} \rightarrow \mathrm{T}$ & $27.90 \%$ & $\mathrm{R} 109 \mathrm{~L}(\mathrm{CGC} \rightarrow \mathrm{CTC}) \ddagger$ & rho $\rightarrow$ & transcription termination factor Rho & - \\
\hline FL(D) & $39,67,876$ & $\mathrm{C} \rightarrow \mathrm{T}$ & $27.90 \%$ & $\mathrm{R} 109 \mathrm{R}(\mathrm{CGC} \rightarrow \mathrm{CGT}) \ddagger$ & rho $\rightarrow$ & transcription termination factor Rho & - \\
\hline FL(D) & $30,14,614$ & $\mathrm{~T} \rightarrow \mathrm{G}$ & $10.00 \%$ & $\mathrm{~N} 253 \mathrm{~T}(\mathrm{AAC} \rightarrow \mathrm{ACC})$ & yqeB $\leftarrow$ & XdhC-CoxI family protein YqeB & - \\
\hline FS(A) & $39,39,901$ & $\mathrm{C} \rightarrow \mathrm{T}$ & $100 \%$ & $\mathrm{~A} 181 \mathrm{~V}(\mathrm{GCC} \rightarrow \mathrm{GTC})$ & $\mathrm{rbsR} \rightarrow$ & $\begin{array}{l}\text { DNA-binding transcriptional dual } \\
\text { regulator RbsR }\end{array}$ & Thymidine metabolism \\
\hline $\mathrm{FS}(\mathrm{A})$ & $41,25,434$ & $\Delta 3 \mathrm{bp}$ & $100 \%$ & coding (154-156/1026 nt) & cytR $\leftarrow$ & $\begin{array}{l}\text { DNA-binding transcriptional repressor } \\
\text { CytR }\end{array}$ & Thymidine metabolism \\
\hline $\mathrm{FS}(\mathrm{A})$ & $23,77,019$ & $\mathrm{~T} \rightarrow \mathrm{G}$ & $16.90 \%$ & $\mathrm{~T} 26 \mathrm{P}(\mathrm{ACC} \rightarrow \mathrm{CCC})$ & menC $\leftarrow$ & o-succinylbenzoate synthase & - \\
\hline $\mathrm{FS}(\mathrm{A})$ & $17,10,565$ & $\mathrm{~A} \rightarrow \mathrm{G}$ & $14.20 \%$ & $\mathrm{E} 579 \mathrm{E}(\mathrm{GAA} \rightarrow \mathrm{GAG})$ & $\mathrm{rsxC} \rightarrow$ & $\begin{array}{l}\text { SoxR }[2 \mathrm{Fe}-2 \mathrm{~S}] \text { reducing system protein } \\
\text { RsxC }\end{array}$ & - \\
\hline $\mathrm{FS}(\mathrm{A})$ & $17,10,904$ & $\mathrm{~A} \rightarrow \mathrm{C}$ & $12.90 \%$ & A692A $(\mathrm{GCA} \rightarrow \mathrm{GCC})$ & $\mathrm{rsxC} \rightarrow$ & $\begin{array}{l}\text { SoxR }[2 \mathrm{Fe}-2 \mathrm{~S}] \text { reducing system protein } \\
\text { RsxC }\end{array}$ & - \\
\hline FS(D) & $41,24,694$ & $\mathrm{G} \rightarrow \mathrm{T}$ & $100 \%$ & P299Q (CCG $\rightarrow$ CAG) & cytR $\leftarrow$ & $\begin{array}{l}\text { DNA-binding transcriptional repressor } \\
\text { CytR }\end{array}$ & Thymidine metabolism \\
\hline FS(D) & $41,87,795$ & $\mathrm{~A} \rightarrow \mathrm{C}$ & $100 \%$ & E438A (GAA $\rightarrow$ GCA) & rpoC $\rightarrow$ & RNA polymerase subunit beta' & \\
\hline $\mathrm{FS}(\mathrm{D})$ & $33,08,226$ & $\mathrm{~T} \rightarrow \mathrm{G}$ & $18.70 \%$ & $\mathrm{~N} 256 \mathrm{~T}(\mathrm{AAC} \rightarrow \mathrm{ACC})$ & deaD $\leftarrow$ & ATP-dependent RNA helicase DeaD & \\
\hline $\mathrm{FS}(\mathrm{D})$ & $20,83,798$ & $\Delta 1 \mathrm{bp}$ & $16.90 \%$ & intergenic (-117/-92) & $\mathrm{dacD} \leftarrow / \rightarrow \mathrm{sbcB}$ & $\begin{array}{l}\text { D-alanyl-D-alanine carboxypeptidase } \\
\text { DacD/exodeoxyribonuclease I }\end{array}$ & \\
\hline
\end{tabular}


Table S9 (continued)

\begin{tabular}{|c|c|c|c|c|c|c|c|}
\hline Population & Position & Mutation & Frequency & Annotation & Locus/Region & Region description & Putative link to adaptation \\
\hline $\mathrm{FS}(\mathrm{D})$ & $17,10,572$ & $\mathrm{C} \rightarrow \mathrm{G}$ & $12.90 \%$ & Q582E $(\mathrm{CAA} \rightarrow \mathrm{GAA})$ & $\mathrm{rsxC} \rightarrow$ & $\begin{array}{l}\text { SoxR }[2 \mathrm{Fe}-2 \mathrm{~S}] \text { reducing system protein } \\
\text { RsxC }\end{array}$ & - \\
\hline $\mathrm{FS}(\mathrm{D})$ & $15,54,101$ & $\mathrm{C} \rightarrow \mathrm{A}$ & $11.80 \%$ & intergenic $(+200 /+207)$ & $\mathrm{fdnI} \rightarrow / \leftarrow \mathrm{yddM}$ & $\begin{array}{l}\text { formate dehydrogenase N subunit } \\
\text { gamma/putative DNA-binding } \\
\text { transcriptional regulator YddM }\end{array}$ & - \\
\hline $\mathrm{FS}(\mathrm{D})$ & $17,10,904$ & $\mathrm{~A} \rightarrow \mathrm{C}$ & $11.00 \%$ & A692A $(\mathrm{GCA} \rightarrow \mathrm{GCC})$ & $\mathrm{rsxC} \rightarrow$ & $\begin{array}{l}\text { SoxR }[2 \mathrm{Fe}-2 \mathrm{~S}] \text { reducing system protein } \\
\text { RsxC }\end{array}$ & - \\
\hline $\mathrm{FS}(\mathrm{D})$ & $25,71,607$ & $\mathrm{~A} \rightarrow \mathrm{C}$ & $10.40 \%$ & L426R $(\mathrm{CTG} \rightarrow \mathrm{CGG})$ & eutE $\leftarrow$ & $\begin{array}{l}\text { putative aldehyde dehydrogenase, } \\
\text { ethanolamine utilization protein }\end{array}$ & - \\
\hline $\mathrm{FS}(\mathrm{E})$ & $22,22,617$ & $\mathrm{G} \rightarrow \mathrm{C}$ & $67.50 \%$ & R169G $(\mathrm{CGT} \rightarrow \mathrm{GGT})$ & $\operatorname{bglX} \leftarrow$ & $\begin{array}{l}\text { beta-D-glucoside glucohydrolase, } \\
\text { periplasmic }\end{array}$ & - \\
\hline $\mathrm{FS}(\mathrm{E})$ & $3,939,469: 1$ & $+\mathrm{T}$ & $54.40 \%$ & coding (110/993 nt) & rbsR $\rightarrow$ & $\begin{array}{l}\text { DNA-binding transcriptional dual } \\
\text { regulator RbsR }\end{array}$ & Thymidine metabolism \\
\hline $\mathrm{FS}(\mathrm{E})$ & $36,59,368$ & IS5 (-) +4 bp & $18.40 \%$ & intergenic (-181/-128) & $\operatorname{arrS} \leftarrow / \rightarrow \operatorname{gadE}$ & $\begin{array}{l}\text { small regulatory RNA } \\
\text { ArrS/DNA-binding transcriptional } \\
\text { activator GadE }\end{array}$ & Thymidine metabolism \\
\hline $\mathrm{FS}(\mathrm{E})$ & $1,26,126$ & $\mathrm{G} \rightarrow \mathrm{A}$ & $12.90 \%$ & $\mathrm{~K} 144 \mathrm{~K}(\mathrm{AAG} \rightarrow \mathrm{AAA})$ & aceF $\rightarrow$ & pyruvate dehydrogenase, E2 subunit & - \\
\hline $\mathrm{FS}(\mathrm{E})$ & $1,07,554$ & $\mathrm{G} \rightarrow \mathrm{T}$ & $10.40 \%$ & intergenic $(+80 /-151)$ & $\operatorname{lpxC} \rightarrow / \rightarrow \operatorname{secM}$ & $\begin{array}{l}\text { UDP-3-O-acyl-N-acetylglucosamine } \\
\text { deacetylase/SecA translation regulator }\end{array}$ & - \\
\hline
\end{tabular}




\section{References}

879 1. Bono, Gensel, C. L., Pfennig, D. W. \& Burch, C. L. Competition and the origins of novelty: experimental evolution of niche-width expansion in a virus. Biol. Lett. 9, 20120616 (2013).

2. Duffy, S., Turner, P. E. \& Burch, C. L. Pleiotropic Costs of Niche Expansion in the RNA Bacteriophage Ф6. Genetics 172, 751-757 (2006).

3. Duffy, S., Burch, C. L. \& Turner, P. E. Evolution of Host Specificity Drives

4. Bono, Gensel, C. L., Pfennig, D. W. \& Burch, C. L. Evolutionary rescue and the coexistence of generalist and specialist competitors: an experimental test. Proc. $R$. Soc. B Biol. Sci. 282, 20151932 (2015).

5. Bono, L. M., Smith, L. B., Pfennig, D. W. \& Burch, C. L. The emergence of performance trade-offs during local adaptation: insights from experimental evolution. Mol. Ecol. 26, 1720-1733 (2017).

6. Sackman, A. M. \& Rokyta, D. R. No Cost of Complexity in Bacteriophages Adapting to a Complex Environment. Genetics 212, 267-276 (2019).

7. Velicer, G. J. \& Lenski, R. E. Evolutionary Trade-Offs Under Conditions of Resource

8. Kassen, R. \& Bell, G. Experimental evolution in Chlamydomonas. IV. Selection in environments that vary through time at different scales. Heredity 80, 732-741 (1998).

898 9. Sacristán, S., Fraile, A., Malpica, J. M. \& García-Arenal, F. An Analysis of Host Adaptation and Its Relationship with Virulence in Cucumber mosaic virus. Phytopathology 95, 827-833 (2005). 
10. Vasilakis, N. et al. Mosquitoes Put the Brake on Arbovirus Evolution: Experimental Evolution Reveals Slower Mutation Accumulation in Mosquito Than Vertebrate Cells. PLOS Pathog. 5, e1000467 (2009).

11. Bennett, A. F. \& Lenski, R. E. Experimental Evolution and Its Role in Evolutionary Physiology. Integr. Comp. Biol. 39, 346-362 (1999).

12. Ostrowski, E. A., Rozen, D. E. \& Lenski, R. E. Pleiotropic Effects of Beneficial Mutations in Escherichia Coli. Evolution 59, 2343-2352 (2005).

13. Hughes, B. S., Cullum, A. J. \& Bennett, A. F. EVOLUTIONARY ADAPTATION TO ENVIRONMENTAL pH IN EXPERIMENTAL LINEAGES OF ESCHERICHIA COLI. Evolution 61, 1725-1734 (2007).

14. Bennett, A. F. \& Lenski, R. E. An experimental test of evolutionary trade-offs during temperature adaptation. Proc. Natl. Acad. Sci. 104, 8649-8654 (2007).

15. Satterwhite, R. S. \& Cooper, T. F. Constraints on adaptation of Escherichia coli to mixed-resource environments increase over time. Evolution 69, 2067-2078 (2015).

16. Karve, S. M., Bhave, D., Nevgi, D. \& Dey, S. Escherichia coli populations adapt to complex, unpredictable fluctuations by minimizing trade-offs across environments. $J$. Evol. Biol. 29, 2545-2555 (2016).

17. Karve, S. M., Bhave, D. \& Dey, S. Extent of adaptation is not limited by unpredictability of the environment in laboratory populations of Escherichia coli. $J$. Evol. Biol. 31, 1420-1426 (2018).

18. Nidelet, T. \& Kaltz, O. Direct and Correlated Responses to Selection in a HostParasite System: Testing for the Emergence of Genotype Specificity. Evolution 61, 1803-1811 (2007). 
19. Roemhild, R., Barbosa, C., Beardmore, R. E., Jansen, G. \& Schulenburg, H. Temporal variation in antibiotic environments slows down resistance evolution in pathogenic Pseudomonas aeruginosa. Evol. Appl. 8, 945-955 (2015).

20. Buckling, A., Kassen, R., Bell, G. \& Rainey, P. B. Disturbance and diversity in experimental microcosms. Nature 408, 961 (2000).

21. Friman, V.-P. \& Buckling, A. Effects of predation on real-time host-parasite coevolutionary dynamics. Ecol. Lett. 16, 39-46 (2013).

22. Buckling, A., Brockhurst, M. A., Travisano, M. \& Rainey, P. B. Experimental adaptation to high and low quality environments under different scales of temporal

23. Jasmin, J.-N. \& Kassen, R. On the experimental evolution of specialization and diversity in heterogeneous environments. Ecol. Lett. 10, 272-281 (2007).

24. Jasmin, J.-N. \& Kassen, R. Evolution of a single niche specialist in variable environments. Proc. R. Soc. Lond. B Biol. Sci. 274, 2761-2767 (2007).

26. Jasmin, J.-N. \& Zeyl, C. Evolution of pleiotropic costs in experimental populations. J.

28. Chen, P. \& Zhang, J. Antagonistic pleiotropy conceals molecular adaptations in

27. Jerison, E. R., Nguyen Ba, A. N., Desai, M. M. \& Kryazhimskiy, S. Chance and necessity in the pleiotropic consequences of adaptation for budding yeast. Nat. Ecol. Evol. 4, 601-611 (2020). changing environments. Nat. Ecol. Evol. 4, 461-469 (2020). 
947 29. Ketola, T. \& Saarinen, K. Experimental evolution in fluctuating environments: tolerance measurements at constant temperatures incorrectly predict the ability to tolerate fluctuating temperatures. J. Evol. Biol. 28, 800-806 (2015).

950 30. Ciota, A. T., Payne, A. F., Ngo, K. A. \& Kramer, L. D. Consequences of in vitro host shift for St. Louis encephalitis virus. J. Gen. Virol. 95, 1281-1288 (2014).

31. Cohen, J. Statistical power analysis for the behavioral sciences. (L. Erlbaum Associates, 1988).

954 32. Chavhan, Y., Malusare, S. \& Dey, S. Larger bacterial populations evolve heavier $736(2020)$. 